\title{
Hyperelliptic Reduction of the Benney Moment Equations
}

\author{
Sadie Baldwin \\ John Gibbons \\ Imperial College \\ 180 Queen's Gate \\ London SW7 2BZ \\ E-mail: sadie.baldwin@imperial.ac.uk, j.gibbons@imperial.ac.uk
}

\begin{abstract}
We consider $N$-parameter reductions of the Benney moment equations. These were shown in Gibbons and Tsarev (1996 Phys. Lett. A 211 19, 1999 Phys. Lett. A 258263 ) to correspond to $N$-parameter families of conformal maps and to satisfy a particular system of PDE. A specific known example of this, the $(N=2)$ elliptic reduction (L Yu and J Gibbons 2000 Inverse Problems 16 605 ) is described. We then consider an analogous reduction for a genus 2 hyperelliptic curve $(N=3)$. The mapping function is given by the inversion of a 2nd kind Abelian integral on the $\Theta$-divisor. This is found explicitly following a method given by Enolskii, Pronine and Richter (2003 J. Nonlinear Science 13 157).
\end{abstract}




\section{Introduction}

\subsection{The Benney Moment Equations}

In 1973, Benney considered an approximation for the two-dimensional equations of motion of an incompressible perfect fluid under a gravitational force [3]. By assuming that the average wave height was small compared to the wavelength he obtained the following equations of motion:

$$
\begin{aligned}
& u_{t}+u u_{x}-\int_{0}^{y} u_{x}\left(x, y^{\prime}, t\right) \mathrm{d} y^{\prime} u_{y}+h_{x}=0, \\
& h_{t}+u h_{x}+\int_{0}^{h} u_{x}\left(x, y^{\prime}, t\right) \mathrm{d} y^{\prime} u_{y}=0
\end{aligned}
$$

where gravity is taken as unity, $u(x, y, t)$ is the horizontal velocity and $h(x, t)$ the height of the free surface. He then showed that if moments are defined by:

$$
A_{n}(x, t)=\int_{0}^{h} u^{n} \mathrm{~d} y,
$$

they satisfy an infinite set of equations

$$
\frac{\partial A_{n}}{\partial t}+\frac{\partial A_{n+1}}{\partial x}+n A_{n-1} \frac{\partial A_{0}}{\partial x}=0 \quad(n=1,2, \ldots)
$$

now called the Benney moment equations. This set is an example of a system of hydrodynamic type; these are defined as equations of the form

$$
\mathbf{u}_{t}+\mathrm{V} \mathbf{u}_{x}=0
$$

where $\mathbf{u}$ is a column vector of variables $\left(u_{1}, u_{2}, \ldots, u_{N}\right)$ and $\mathrm{V}$ is an $N$ by $N$ matrix depending on $\mathbf{u}$ [5]. In the case of Benney's equations, however, $N$ is infinite.

Identical moment equations can alternatively be derived from a Vlasov equation [8], $[16]$

$$
\frac{\partial f}{\partial t_{2}}+p \frac{\partial f}{\partial x}-\frac{\partial A_{0}}{\partial x} \frac{\partial f}{\partial p}=0
$$

Here $f=f(x, p, t)$ is a distribution function and the moments are defined instead by

$$
A_{n}=\int_{-\infty}^{\infty} p^{n} f \mathrm{~d} p
$$

The equation of motion (4) has the Lie-Poisson structure :

$$
\frac{\partial f}{\partial t}+\left\{f, \frac{\delta H}{\delta f}\right\}_{p, x}=0
$$

where $\{\cdot, \cdot\}_{p, x}$ is the canonical Poisson bracket. Kupershmidt and Manin showed directly that the moment equations are Hamiltonian [12], [13]. If we set $H=\frac{1}{2} H_{2}=$ $\frac{1}{2}\left(A_{2}+A_{0}^{2}\right), \mathbf{A}=\left(A_{0}, A_{1}, \ldots\right)$, then

$$
\frac{\partial \mathbf{A}}{\partial t}=B \frac{\partial H}{\partial \mathbf{A}}
$$


where the matrix operator $B$ is given by

$$
B_{n, m}=n A_{n+m-1} \frac{\partial}{\partial x}+m \frac{\partial}{\partial x} \cdot A_{n+m-1} .
$$

This is consistent with (5) in the sense that if $H$ is some function of the moments, the moment equations resulting from (5) and (6) are identical.

Benney showed in [3] that system (3) has infinitely many conserved densities, polynomial in the $A_{n}$. One of the most direct ways to calculate these is to use generating functions $[12]$.

Let $\lambda(x, p, t)$, a formal series in $p$, be the generating function of the moments

$$
\lambda(x, p, t)=p+\sum_{n=0}^{\infty} \frac{A_{n}}{p^{n+1}}
$$

and let $p(x, \lambda, t)$ be the inverse series

$$
p(x, \lambda, t)=\lambda-\sum_{m=0}^{\infty} \frac{H_{m}}{\lambda^{m+1}} .
$$

We note here that if $A_{n}=\int_{-\infty}^{\infty} p^{n} f \mathrm{~d} p$ is substituted into (7), then this can be understood as the asymptotic series as $p \rightarrow \infty$ of the integral

$$
\lambda_{R}=p+\mathrm{P} \int_{-\infty}^{\infty} \frac{f\left(x, p^{\prime}, t\right)}{\left(p-p^{\prime}\right)} \mathrm{d} p^{\prime}
$$

where $\mathrm{P}$ denotes the principal value.

Comparing the first derivatives of $\lambda(x, p, t)$, we obtain the PDE

$$
\frac{\partial \lambda}{\partial t}+p \frac{\partial \lambda}{\partial x}=\frac{\partial \lambda}{\partial p}\left(\frac{\partial p}{\partial t}+p \frac{\partial p}{\partial x}+\frac{\partial A_{0}}{\partial x}\right)
$$

If we now hold $p$ constant, this gives

$$
\frac{\partial \lambda}{\partial t}+p \frac{\partial \lambda}{\partial x}-\frac{\partial A_{0}}{\partial x} \frac{\partial \lambda}{\partial p}=0
$$

which is a Vlasov equation of the same form as (4). Thus (4) and (10) have the same characteristics. Any function of $\lambda$ and $f$ must satisfy the same equation.

Alternatively, if we hold $\lambda$ constant in (9), then we obtain the conservation equation

$$
\frac{\partial p}{\partial t}+\frac{\partial}{\partial x}\left(\frac{1}{2} p^{2}+A_{0}\right)=0
$$

Substituting the formal series of $p(x, \lambda, t)$ into (11), we see that each $H_{n}$ is polynomial in the $A_{n}$ and is a conserved density. Any of the $H_{n}$ could therefore be used as the Hamiltonian in (5). From this we define the Benney hierarchy to be the family of evolution equations

$$
\frac{\partial f}{\partial t_{n}}+\left\{f, \frac{1}{n} \frac{\delta H_{n}}{\delta f}\right\}=0 .
$$

We note that $\lambda$ satisfies an equation analogous to this,

$$
\frac{\partial \lambda}{\partial t_{n}}+\left\{\lambda, \frac{1}{n} \frac{\delta H_{n}}{\delta f}\right\}=0
$$


and that the Hamiltonians satisfy the relation

$$
\left(\frac{1}{n} \frac{\delta H_{n}}{\delta f}\right)=\left(\frac{\lambda^{n}}{n}\right)_{+}
$$

where $(\cdot)_{+}$denotes the polynomial part of the expansion. It was shown in [13] that these Hamiltonians Poisson commute.

\subsection{Reductions of the moment equations}

Suppose that for some point $p=\hat{p}_{i}, \lambda\left(\hat{p}_{i}\right)=\hat{\lambda}_{i}$

$$
\left.\frac{\partial \lambda}{\partial p}\right|_{p=\hat{p}_{i}}=0
$$

then (9) reduces to:

$$
\frac{\partial \hat{\lambda}_{i}}{\partial t}+\hat{p}_{i} \frac{\partial \hat{\lambda}_{i}}{\partial x}=0
$$

where $\frac{\partial \hat{\lambda}_{i}}{\partial t}=\left.\frac{\partial \lambda}{\partial t}\right|_{p=\hat{p}_{i}}$ and $\frac{\partial \hat{\lambda}_{i}}{\partial x}=\left.\frac{\partial \lambda}{\partial x}\right|_{p=\hat{p}_{i}}$. We say that $\hat{\lambda}_{i}$ is a Riemann invariant with characteristic speed $\hat{p}_{i}$.

A hydrodynamic type system with $N$ independent variables can not in general, for $N \geq 3$, be expressed in terms of Riemann invariants. If such a system does have $N$ Riemann invariants, it is called diagonalisable. Tsarev showed in [14] that if a diagonal hydrodynamic-type system

$$
\frac{\partial \hat{\lambda}_{i}}{\partial t}+\mathrm{v}_{i}(\hat{\lambda}) \frac{\partial \hat{\lambda}_{i}}{\partial x}=0 \quad(i=1,2, \ldots, N) .
$$

is semi-Hamiltonian, that is if

$$
\partial_{j}\left(\frac{\partial_{i} \mathrm{v}_{k}}{\mathrm{v}_{i}-\mathrm{v}_{k}}\right)=\partial_{i}\left(\frac{\partial_{j} \mathrm{v}_{k}}{\mathrm{v}_{j}-\mathrm{v}_{k}}\right), \quad i \neq j \neq k,
$$

for $i, j, k$ distinct, where

$$
\partial_{k}=\frac{\partial}{\partial \hat{\lambda}_{k}}
$$

then it can be solved by the hodograph transformation. Any Hamiltonian system of hydrodynamic type is semi-Hamiltonian. Given a second equation of type (12)

$$
\frac{\partial \hat{\lambda}_{i}}{\partial \tau}+\mathrm{w}_{i}(\hat{\lambda}) \frac{\partial \hat{\lambda}_{i}}{\partial x}=0 \quad(i=1,2, \ldots, N),
$$

and requiring it to be consistent with (12), we find that the $\mathrm{w}_{i}(\hat{\lambda})$ satisfy the overdetermined linear system

$$
\frac{\partial_{k} \mathrm{w}_{i}}{\mathrm{w}_{i}-\mathrm{w}_{k}}=\frac{\partial_{k} \mathrm{v}_{i}}{\mathrm{v}_{i}-\mathrm{v}_{k}}, \quad i \neq k
$$

These equations are consistent provided (12) is semi-Hamiltonian. If the condition (14) holds, we say that (12) and (13) commute. In this case a set of equations for the unknowns $\hat{\lambda}_{i}(x, t)$ is given by :

$$
\mathrm{w}_{i}(\hat{\lambda})=\mathrm{v}_{i}(\hat{\lambda}) t+x, \quad(i=1,2, \ldots, N)
$$


where $t$ and $x$ are the independent variables. Thus any reduction of this type can be solved in principle.

This generalized hodograph construction cannot easily be applied directly to the Benney equations, as these have infinitely many dependent variables. However we will now consider families of distribution functions $f$, which are parameterised by finitely many $N$ Riemann invariants $\hat{\lambda}_{i}(x, t)$. We are interested in the case [9], [10] where the function $\lambda(p, x, t)$ is such that only $N$ of the moments are independent. Then there are $N$ characteristic speeds, assumed real and distinct, and $N$ corresponding Riemann invariants $\left(\hat{p}_{i}, \hat{\lambda}_{i}\right)$, so Benney's equations reduce to a diagonal system of hydrodynamic type with finitely many dependent variables $\hat{\lambda}_{i}$,

$$
\frac{\partial \hat{\lambda}_{i}}{\partial t}+\hat{p}_{i}(\hat{\lambda}) \frac{\partial \hat{\lambda}_{i}}{\partial x}=0 \quad(i=1,2, \ldots, N) .
$$

Such a system is called a reduction of Benney's equations.

Zakharov found one such reduction by dividing the fluid flow into $K$ horizontal layers each with $y$-independent horizontal velocity $u_{i}$ and depth $h_{i}$ [16]. The system (2) is therefore reduced to the set of $2 K$ equations

$$
\begin{aligned}
& h_{i_{t}}+\left(u_{i} h_{i}\right)_{x}=0, \\
& u_{i_{t}}+u_{i} u_{i_{x}}+\sum_{i=1}^{N} h_{i_{x}}=0, \quad(i=1,2, \ldots, K),
\end{aligned}
$$

with moments

$$
A_{n}=\sum_{i=0}^{K} h_{i} u_{i}^{n} .
$$

This is clearly a system of hydrodynamic type dependent on $2 K$ variables. Here, the generating function $\lambda$ is given by

$$
\lambda=p+\sum_{i=1}^{K} \frac{h_{i}}{p-u_{i}} .
$$

The construction of a more general family of solutions for equations of this type was outlined in [9] and [10]. Instead of considering the principal value integral (7), we now define a new function $\lambda_{+}(x, p, t)$ :

$$
\lambda_{+}(x, p, t)=p+\int_{\Lambda} \frac{f\left(x, p^{\prime}, t\right)}{p-p^{\prime}} \mathrm{d} p^{\prime}
$$

where $\Lambda$ is an indented contour passing below the point $p$. This has the same asymptotics as $\lambda(x, p, t)$, provided all the moments $A_{n}$ exist, and it can be analytically continued into the upper half of the $p$-plane. If $f$ satisfies a Hölder condition on the real $p$-axis [11], that is if $\exists \mu, \gamma>0$ such that

$$
|f(x, p, t)-f(x, q, t)| \leq \mu|p-q|^{\gamma}
$$

for $q, \operatorname{Im}(q)>0$, sufficiently close to $p \in \mathbb{R}$, then the boundary value of $\lambda_{+}$satisfies

$$
\lambda_{+}=\lambda_{R}-i \pi f
$$


on $\mathbb{R}$.

An elementary example is the case where the map $\lambda_{+}$takes the upper half $p$-plane to the upper half $\lambda$-plane with a vertical slit as follows.

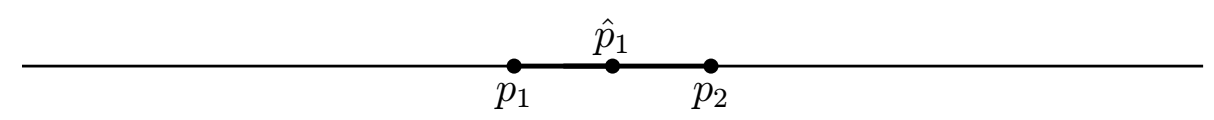

Figure 1. The p-plane.

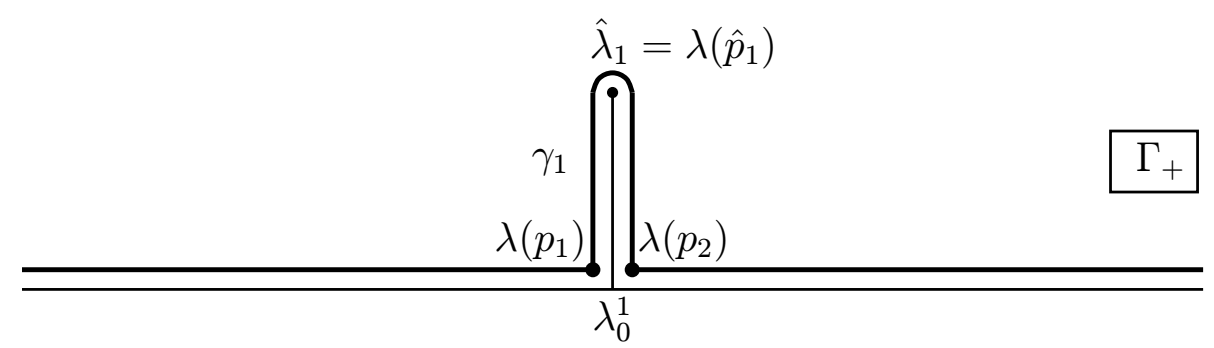

Figure 2. The $\lambda$-plane.

This is a Schwarz-Christoffel map:

$$
\lambda_{+}(x, p, t)=p+\int_{\infty}^{p} \frac{p^{\prime}-\hat{p}_{1}}{\sqrt{\left(p^{\prime}-p_{1}\right)\left(p^{\prime}-p_{2}\right)}} \mathrm{d} p^{\prime} .
$$

If the residue at infinity is set to be zero, then this imposes the condition $\hat{p}_{1}=\frac{1}{2}\left(p_{1}+p_{2}\right)$ and we get solution

$$
\begin{aligned}
\lambda_{+}(x, p, t) & =\hat{p}_{1}+\sqrt{p^{2}-\left(p_{1}+p_{2}\right) p+p_{1} p_{2}} \\
& =\hat{p}_{1}+\sqrt{\left(p-\hat{p}_{1}\right)^{2}+2 A_{0}}
\end{aligned}
$$

(from the expansion as $p \rightarrow \infty$ ). This gives a time independent solution of Benney's equations (5)

$$
\frac{\partial f}{\partial t}+\left\{f, \frac{1}{2} p^{2}+A_{0}\right\}_{p, x}=0 .
$$

The two parameters $p_{1}$ and $p_{2}$ are not independent, as for consistency their sum must be a constant. Hence only the end point of the slit in the $\lambda$-plane is variable. This is the Riemann invariant.

The above construction may be generalized. Suppose the relation $f=F\left(\lambda_{R}\right)$ holds in some region of the $(x, p)$-plane at some time $t$. Then since both (4) and (10) have the same characteristics, the relation will be preserved by the dynamics. In this case the definition for $\lambda_{+}$(16) becomes a nonlinear singular integral equation:

$$
\lambda_{+}(x, p, t)=p+\int_{\Lambda} \frac{F\left(\lambda_{R}\left(x, p^{\prime}, t\right)\right)}{p-p^{\prime}} \mathrm{d} p^{\prime}
$$


The solutions to (17) can be described in terms of a conformal mapping of a slit domain. We take the upper half $\lambda$-plane, $\Gamma_{+}$, and draw a Jordan $\operatorname{arc} c$ in $\Gamma_{+}$starting from a point, $\lambda_{0}^{1}$, on the real axis. We then fix an arbitrary point on this arc, $\hat{\lambda}_{1}$, and make a slit $\gamma_{1}$ running along the arc from $\lambda_{0}^{1}$ to $\hat{\lambda}_{1}$.

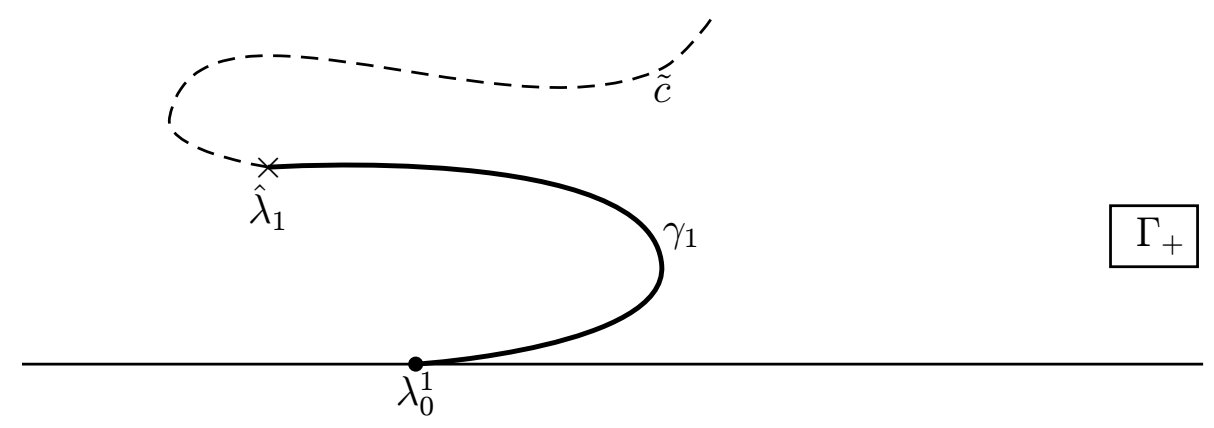

Figure 3. The slit $\gamma_{1}$ on the Jordan $\operatorname{arc} c=\gamma_{1} \cup \tilde{c}$.

Note that the slit $\gamma_{1}$ is given by the relation

$$
\operatorname{Im}\left(\lambda_{+}\right)=-\pi F\left(\operatorname{Re}\left(\lambda_{+}\right)\right)
$$

and so $F$ must be continuous with $F \leq 0$. The function $p\left(\lambda_{+}, \hat{\lambda}_{1}\right)$ is then determined uniquely by the following properties.

(i) $p\left(\lambda_{+}, \hat{\lambda}_{1}\right)$ has a branch point at $\hat{\lambda}_{1}$, that is

$$
p \sim \hat{p}+c\left(\lambda-\hat{\lambda}_{1}\right)^{\frac{1}{2}}+O\left(\lambda-\hat{\lambda}_{1}\right) .
$$

(ii) $p\left(\lambda_{+}, \hat{\lambda}_{1}\right)$ is real on the real $\lambda_{+}$-axis and on both sides of $\gamma_{1}$.

(iii) $p\left(\lambda_{+}, \hat{\lambda}_{1}\right)$ is analytic in the cut half plane $\Gamma_{+}$.

(iv) As $|\lambda| \rightarrow \infty$, with $\operatorname{Im}\left(\lambda_{+}\right) \geq 0, p\left(\lambda_{+}, \hat{\lambda}_{1}\right)$ has the expansion

$$
p\left(\lambda_{+}, \hat{\lambda}_{1}\right) \sim \lambda_{+}+O\left(\frac{1}{\lambda_{+}}\right) .
$$

The evolution of $p$ is then given by (11); expanding near $\hat{\lambda}_{1}$ gives:

$$
\frac{\partial \hat{\lambda}_{1}}{\partial t}+\hat{p} \frac{\partial \hat{\lambda}_{1}}{\partial x}=0
$$

Thus $\hat{\lambda}_{1}$ is a Riemann invariant with characteristic speed $\hat{p}=p\left(\hat{\lambda}_{1}\right)$.

It is possible to generalize this construction further to $N$ non-intersecting slits. Here, each of the slits $\gamma_{i}$ is made along a fixed path starting on the real $\lambda_{+}$-axis and ending in a branch point $\hat{\lambda}_{i}$.

Again, $\hat{\lambda}_{i}$ are the Riemann invariants of the system with associated characteristic speeds $p\left(\hat{\lambda}_{i}\right)$ and the slits $\gamma_{i}$ are given by

$$
\operatorname{Im}\left(\lambda_{+}\right)=-\pi F_{i}\left(\operatorname{Re}\left(\lambda_{+}\right)\right)
$$

where $F_{i} \leq 0$ are continuous functions. 


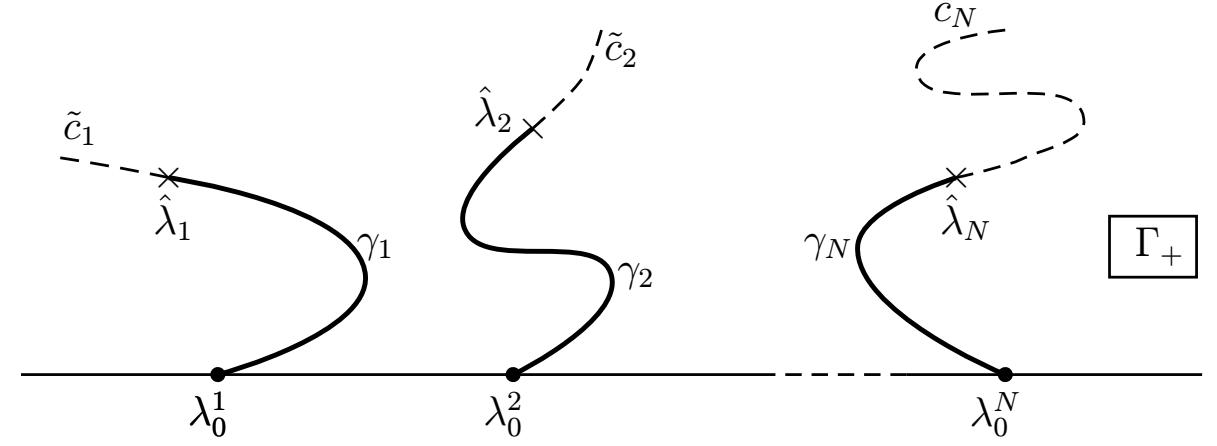

Figure 4. $N$ slits on $\Gamma_{+}$. The slit $\gamma_{i}$ is on the Jordan $\operatorname{arc} c_{i}=\gamma_{i} \cup \tilde{c}_{i}$.

\section{Elliptic reduction}

A specific example of a reduction of the Benney moment equations, the elliptic reduction, was considered by $\mathrm{Yu}$ and Gibbons in [15].
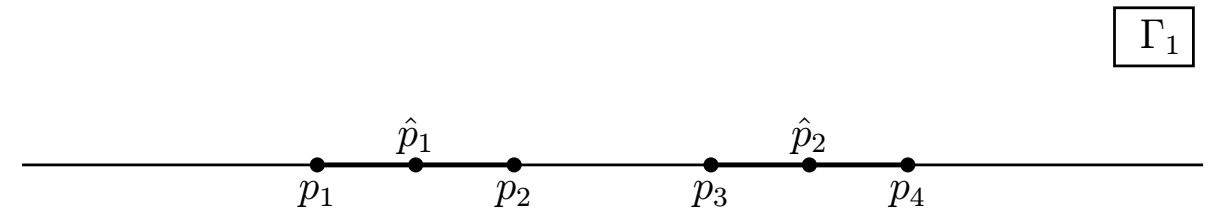

Figure 5. The $p$-plane for the elliptic reduction.

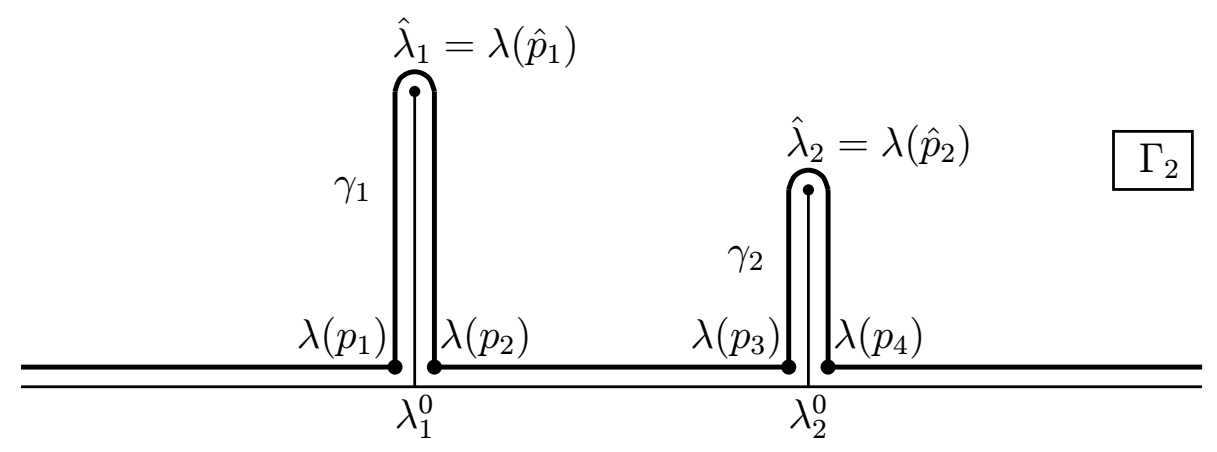

Figure 6. The $\lambda$-plane for the elliptic reduction.

The upper half $p$-plane is mapped into a polygonal domain in the upper half $\lambda$-plane. Consequently, $\lambda(p): \Gamma_{1} \rightarrow \Gamma_{2}$ is again of Schwarz-Christoffel type:

$$
\lambda(p)=p+\int_{-\infty}^{p}\left[\varphi\left(p^{\prime}\right)-1\right] d p^{\prime}
$$

where $\varphi(p)$ is given by:

$$
\varphi(p)=\frac{\prod_{i=1}^{2}\left(p-\hat{p}_{i}\right)}{\sqrt{\prod_{i=1}^{4}\left(p-p_{i}\right)}}=\frac{p^{2}-\alpha p-\beta}{\sqrt{\prod_{i=1}^{4}\left(p-p_{i}\right)}} .
$$


The two regions in figures 5 and 6 are defined as follows. $\Gamma_{1}$ is the upper half complex $p$-plane with six points marked on the real axis, $p_{1}<\hat{p}_{1}<p_{2}<p_{3}<\hat{p}_{2}<p_{4}$, and $\Gamma_{2}$ is the upper half $\lambda$-plane with two vertical slits $\gamma_{i}$ running from a fixed point $\lambda_{i}^{0}$ on the real axis to $\hat{\lambda}_{i}^{0}$, which is variable. By imposing the conditions

$$
\begin{aligned}
& \lambda\left(p_{1}\right)=\lambda\left(p_{2}\right)=\lambda_{1}^{0}, \\
& \lambda\left(p_{3}\right)=\lambda\left(p_{4}\right)=\lambda_{2}^{0},
\end{aligned}
$$

where $\lambda_{1}^{0}$ and $\lambda_{2}^{0}$ are prescribed real constants, we obtain the required mapping (figure 6). We note here that, if we define $\mathfrak{b}$ to be a closed loop encircling the interval $\left[p_{1}, p_{2}\right]$ in the positive sense (see figure 7 ), then a consequence of (19) is

$$
\int_{\mathfrak{b}} \varphi(p) \mathrm{d} p=0 .
$$

Another of the four conditions in (19) was then replaced by setting the residue of $\lambda(p)$ at infinity to be zero, that is:

$$
\alpha=\frac{1}{2} \sum_{i=1}^{4} p_{i}
$$

and thus $\varphi(p) \mathrm{d} p$ is a second kind Abelian differential on the elliptic Riemann surface

$$
R_{1}=\left\{(v, p) \in \mathbb{C}^{2}: v^{2}=\prod_{i=1}^{4}\left(p-p_{i}\right)\right\} .
$$

That is, $\varphi(p) \mathrm{d} p$ is a meromorphic 1-form on $R_{1}$ with zero residue at each singular point. We see that the function $\lambda(p)$ depends on 2 parameters, $\operatorname{Im}\left(\hat{\lambda}_{1}\right)$ and $\operatorname{Im}\left(\hat{\lambda}_{2}\right)$ say.

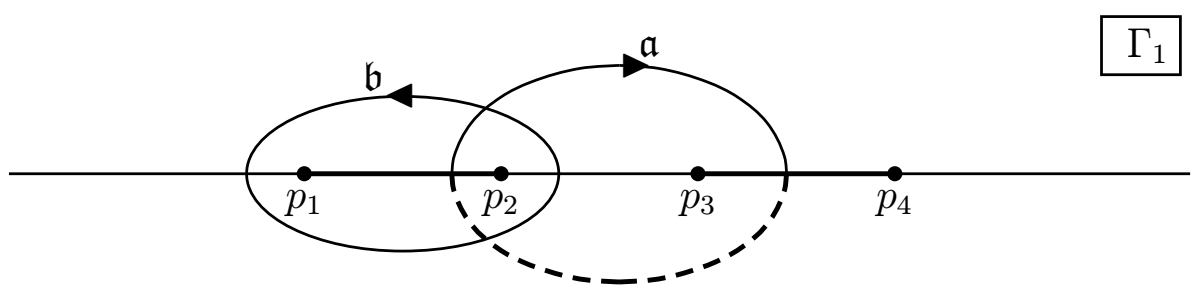

Figure 7. The cycles on the Riemann surface $R_{1}$. The $\mathfrak{b}$-cycle is a closed loop on the first sheet and the $\mathfrak{a}$-cycle is completed on the second sheet (broken line).

The integral (18) was evaluated by first substituting

$$
p=p_{4}-\frac{1}{\wp(\chi)-\wp\left(\chi_{0}\right)}
$$

into $\varphi(p)$ and then expanding the integrand near its singularities. Here, $\wp$ is the Weierstrass elliptic function with half-periods $\omega_{1}, \omega_{2}$ given by

$$
\omega_{1}=\frac{1}{2} \int_{\mathfrak{b}} \frac{1}{\sqrt{\prod_{i=1}^{4}\left(p-p_{i}\right)}} \mathrm{d} p, \quad \omega_{2}=\frac{1}{2} \int_{\mathfrak{a}} \frac{1}{\sqrt{\prod_{i=1}^{4}\left(p-p_{i}\right)}} \mathrm{d} p,
$$


where $\mathfrak{a}$ and $\mathfrak{b}$ are as in figure 7 , and

$$
\wp\left(\chi_{0}\right)=-\sum_{i=1}^{3} \frac{1}{3\left(p_{4}-p_{i}\right)} .
$$

The explicit value of the map $\lambda(p)$ was shown to be:

$$
\lambda(p)=-\frac{1}{\wp^{\prime}\left(\chi_{0}\right)}\left[\zeta\left(\chi+\chi_{0}\right)+\zeta\left(\chi-\chi_{0}\right)+2 \frac{\zeta\left(\omega_{1}\right)}{\omega_{1}} \chi\right]+\lambda_{2}^{0}
$$

\section{Genus 2 hyperelliptic reduction}

Conceptually, the simplest extension of the elliptic reduction is to consider the case where $\lambda(p): \Gamma_{1} \rightarrow \Gamma_{2}$ is as given in figures 8 and 9 .
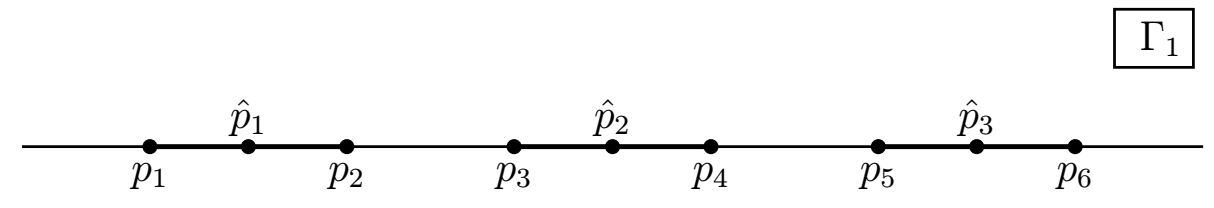

Figure 8. The $p$-plane for the genus 2 hyperelliptic reduction.

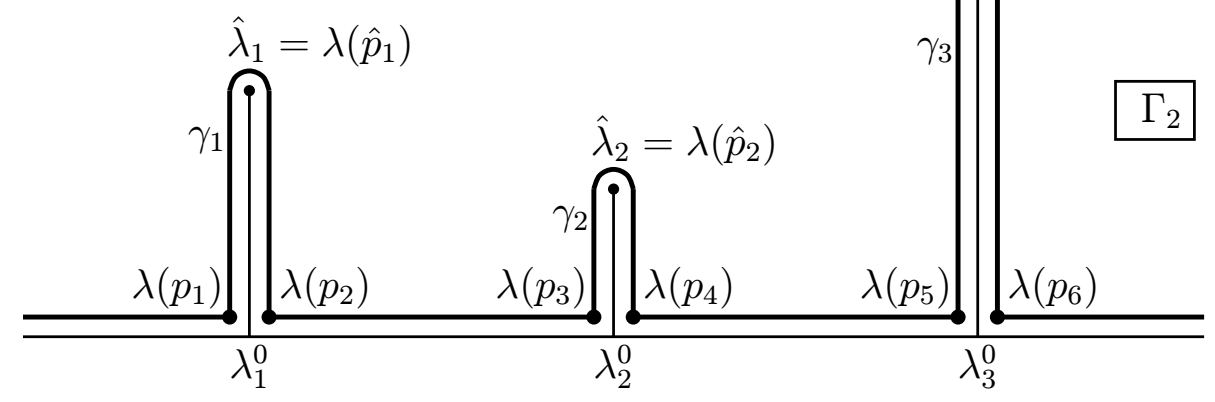

Figure 9. The $\lambda$-plane associated with figure 8 .

Here $\lambda(p)$ is again in Schwartz-Christoffel form:

$$
\lambda(p)=p+\int_{-\infty}^{p}\left[\varphi\left(p^{\prime}\right)-1\right] \mathrm{d} p^{\prime},
$$

where $\varphi$ is given by:

$$
\varphi(p)=\frac{\prod_{i=1}^{3}\left(p-\hat{p}_{i}\right)}{\sqrt{\prod_{i=1}^{6}\left(p-p_{i}\right)}} .
$$

This mapping takes the region $\Gamma_{1}$, the upper half complex $p$-plane with nine points marked on the real axis

$$
p_{1}<\hat{p}_{1}<p_{2}<p_{3}<\hat{p}_{2}<p_{4}<p_{5}<\hat{p}_{3}<p_{6},
$$


to $\Gamma_{2}$, the upper half complex $\lambda$-plane with three vertical slits going from the fixed points $\lambda_{i}^{0}$ to the variable points $\hat{\lambda}_{i},(i=1,2,3)$.

The conditions imposed in the elliptic case are now extended as follows. We require that $\lambda(p)=p+O\left(\frac{1}{p}\right)$ as $p \rightarrow \infty$ and that

$$
\lambda\left(p_{2 n-1}\right)=\lambda\left(p_{2 n}\right)=\lambda_{n}^{0} \quad(n=1,2,3)
$$

where $\lambda_{n}^{0}$ are prescribed real constants. This means that $\lambda(p)$ is a function of 3 independent parameters which may be taken to be $\operatorname{Im}\left(\hat{\lambda_{i}}\right)(i=1,2,3)$. We can replace one of these conditions with the constraint that the residue of $\lambda(p)$, as $p \rightarrow \infty$ on either sheet, is zero. Rewriting

$$
\varphi(p)=\frac{p^{3}-\alpha p^{2}-\beta p-\gamma}{\sqrt{\prod_{i=1}^{6}\left(p-p_{i}\right)}},
$$

we find that the expansion of $\varphi(p)$ near infinity is

$$
1+\frac{\left(\frac{1}{2} \sum_{i=1}^{6} p_{i}-\alpha\right)}{p}+O\left(\frac{1}{p^{2}}\right) .
$$

The condition on the residue is thus satisfied when

$$
\alpha=\frac{1}{2} \sum_{i=1}^{6} p_{i}
$$

that is

$$
\sum_{i=1}^{3} \hat{p}_{i}=\frac{1}{2} \sum_{i=1}^{6} p_{i}
$$

It follows that $\varphi(p) \mathrm{d} p$ is a second kind Abelian differential on the Riemann surface $R_{2}$ defined by

$$
v^{2}=\prod_{i=1}^{6}\left(p-p_{i}\right)
$$

This surface can be constructed from 2 copies of the complex $p$-plane joined along the closed intervals $\left[p_{1}, p_{2}\right],\left[p_{3}, p_{4}\right]$ and $\left[p_{5}, p_{6}\right]$. The homology basis $\left(\mathfrak{a}_{1}, \mathfrak{a}_{2} ; \mathfrak{b}_{1}, \mathfrak{b}_{2}\right)$ for the Riemann surface is given in figure 10.

Note that the cycle $\mathfrak{a}_{i}$ only intersects $\mathfrak{b}_{i}$ and that the intersections have cycle index $\mathfrak{a}_{i} \circ \mathfrak{b}_{j}=\delta_{i j}$.

\section{Transformation of the integral}

The integral we need to evaluate, $(22)$ is

$$
\lambda(p)=p+\int_{-\infty}^{p}\left[\frac{\prod_{i=1}^{3}\left(p^{\prime}-\hat{p}_{i}\right)}{\sqrt{\prod_{i=1}^{6}\left(p^{\prime}-p_{i}\right)}}-1\right] \mathrm{d} p^{\prime}
$$




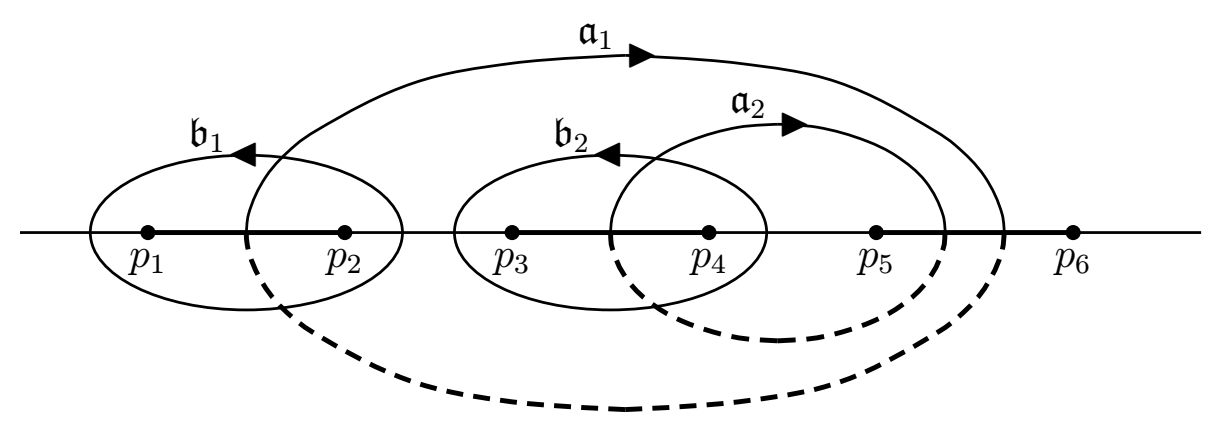

Figure 10. A homology basis on the genus 2 Riemann surface, $R_{2}$. The $\mathfrak{b}$-cycles are closed loops on the first sheet and the a-cycles are completed on the second sheet (broken line).

By substituting $p=p_{6}-\frac{1}{t}$ into the integrand $(\varphi(p)-1) \mathrm{d} p$, we find

$$
\begin{aligned}
(\varphi(p)-1) \mathrm{d} p & =\left(\frac{\prod_{i=1}^{3}\left[\left(p_{6}-\hat{p}_{i}\right) t-1\right]}{\sqrt{\prod_{i=1}^{6}\left[\left(p_{6}-p_{i}\right) t-1\right]}}-1\right) \frac{\mathrm{d} t}{t^{2}} \\
& =\left(\frac{\left(a t^{3}+b t^{2}+c t-1\right)}{\sqrt{\prod_{i=1}^{6}\left[\left(p_{6}-p_{i}\right) t-1\right]}}-1\right) \frac{\mathrm{d} t}{t^{2}}
\end{aligned}
$$

for some constants $a, b, c$. If we now take out the constant factor

$$
k=\left(\frac{-4}{\prod_{i=1}^{5}\left(p_{6}-p_{i}\right)}\right)^{\frac{1}{2}}
$$

from this integrand, then we obtain a standardized form for the irrational denominator:

$$
\varphi(p) \mathrm{d} p=k \frac{a t^{3}+b t^{2}+c t-1}{s} \frac{\mathrm{d} t}{t^{2}}
$$

where

$$
s^{2}=\left(4 t^{5}+\mu_{4} t^{4}+\mu_{3} t^{3}+\mu_{2} t^{2}+\mu_{1} t+\mu_{0}\right) .
$$

We note here that the constant $\mu_{0}$ is equal to $k^{2}$. Thus

$$
\begin{aligned}
\varphi(p) \mathrm{d} p & =k\left(\frac{a t^{3}+b t^{2}+c t-1}{s}\right) \frac{\mathrm{d} t}{t^{2}}, \\
& =k\left(a t+b+\frac{c}{t}-\frac{1}{t^{2}}\right) \frac{\mathrm{d} t}{s} .
\end{aligned}
$$

Here $\mathrm{d} t / s$ and $(t \mathrm{~d} t) / s$ are a basis of holomorphic Abelian differentials. To evaluate this integral we require a specialized form of the Jacobi inversion theorem. We will begin, though, by outlining the setting for the theorem. 


\section{Abelian integrals}

Let $R(s, t)$, be a hyperelliptic curve where $s$ and $t$ satisfy

$$
s^{2}=4 \prod_{i=1}^{5}\left(t-t_{i}\right)=\sum_{i=0}^{5} \mu_{i} t^{i}
$$

and $\mu_{5}=4$. Following [4], we define a set of holomorphic differentials $\mathrm{d} u_{i}$, and the associated set of second kind differentials $\mathrm{d} r_{i}$. In this genus 2 case these are

$$
\mathrm{d} u_{1}=\frac{1}{s} \mathrm{~d} t, \quad \mathrm{~d} u_{2}=\frac{t}{s} \mathrm{~d} t
$$

and

$$
\mathrm{d} r_{1}=\frac{\mu_{3} t+2 \mu_{4} t^{2}+12 t^{3}}{4 s} \mathrm{~d} t, \quad \mathrm{~d} r_{2}=\frac{t^{2}}{s} \mathrm{~d} t
$$

respectively. From the construction of the canonical homology basis (figure 10) and the fact that the $p_{i}$ are real, it follows that the periods of these two sets of differentials are real around the $\mathfrak{b}$-cycles and imaginary around the $\mathfrak{a}$-cycles. We define the four $2 \times 2$ matrices $\omega, \omega^{\prime}, \eta, \eta^{\prime}$ by

$$
\begin{aligned}
2 \omega_{i j} & =\oint_{\mathfrak{b}_{j}} \mathrm{~d} u_{i}, & 2 \omega_{i j}^{\prime} & =\oint_{\mathfrak{a}_{j}} \mathrm{~d} u_{i}, \\
2 \eta_{i j} & =-\oint_{\mathfrak{b}_{j}} \mathrm{~d} r_{i}, & 2 \eta_{i j}^{\prime} & =-\oint_{\mathfrak{a}_{j}} \mathrm{~d} r_{i}, \quad(i, j=1,2) .
\end{aligned}
$$

As a direct consequence of the Riemann bilinear identity (see [2]), these matrices satisfy the generalized Legendre relation

$$
\left(\begin{array}{cc}
\omega & \omega^{\prime} \\
\eta & \eta^{\prime}
\end{array}\right)\left(\begin{array}{cc}
0 & -1_{2} \\
1_{2} & 0
\end{array}\right)\left(\begin{array}{cc}
\omega & \omega^{\prime} \\
\eta & \eta^{\prime}
\end{array}\right)^{\mathrm{T}}=-\frac{i \pi}{2}\left(\begin{array}{cc}
0 & -1_{2} \\
1_{2} & 0
\end{array}\right),
$$

where $1_{2}$ is the $2 \times 2$ identity matrix. These period matrices, which, apart from a scalar factor, preserve the matrix

$$
\left(\begin{array}{cc}
0 & -1_{2} \\
1_{2} & 0
\end{array}\right)
$$

are given by elements of the Symplectic group $S p(4)$ multiplied by the factor $(-i \pi / 2)^{\frac{1}{2}}$.

Letting $\Gamma=2 \omega \otimes 2 \omega^{\prime}$ be the lattice generated by the periods of the holomorphic differentials, we define the Jacobi variety, $\operatorname{Jac}(R)$, to be the 2 dimensional complex torus $\mathbb{C}^{2} / \Gamma[2]$. The Abel map, $\mathfrak{A}: R \rightarrow \operatorname{Jac}(R)$, is given by $\mathbf{u}(z):$

$$
u_{i}(z)=\int_{z_{0}}^{z} \mathrm{~d} u_{i}, \quad(i=1,2)
$$

where the $u_{i}(z)$ are taken modulo $\Gamma$ and the base point $z_{0}$ is any fixed point in $R$. These create a 1 dimensional image of the hyperelliptic curve in the Jacobi variety.

For the inversion theorem we require an extension of this map to a set of points. 
Definition 5.1 $A$ divisor $\mathcal{D}$ on the Riemann surface $R$ is defined by the finite formal sum

$$
\mathcal{D}=\sum_{i}^{M} n_{i} z_{i}
$$

where $n_{i} \in \mathbb{Z}$ and $z_{i}=\left(s_{i}, t_{i}\right) \in R$.

We define the Abel mapping of $\mathcal{D}$ into $\operatorname{Jac}(R)$ by

$$
\mathfrak{A}(\mathcal{D})=\sum_{i}^{M} n_{i} \int_{z_{0}}^{z_{i}} \mathrm{~d} \mathbf{u} \quad \bmod \Gamma
$$

in genus 2 this is:

$$
\mathfrak{A}(\mathcal{D})=\left(\sum_{i}^{M} n_{i} \int_{z_{0}}^{z_{i}} \mathrm{~d} u_{1}, \sum_{i}^{M} n_{i} \int_{z_{0}}^{z_{i}} \mathrm{~d} u_{2}\right) .
$$

The lower limit of integration here the point $z_{0}$, is called the base point of the Abel map.

\subsection{Hyperelliptic functions (genus 2)}

Definition 5.2 The theta function in genus 2 is defined by the Fourier series

$$
\theta\left((2 \omega)^{-1} \mathbf{u} ; \omega, \omega^{\prime}\right)=\sum_{\mathbf{m} \in \mathbb{Z}^{2}} \exp i \pi\left[\mathbf{m}^{\mathrm{T}} \omega^{-1} \omega^{\prime} \mathbf{m}+2 \mathbf{m}^{\mathrm{T}}(2 \omega)^{-1} \mathbf{u}\right] .
$$

This series converges because $B=\omega^{-1} \omega^{\prime}$ has positive definite imaginary part.

Let $\mathbf{v}$ be the normalized Abel map, $\mathbf{v}=(2 \omega)^{-1} \mathbf{u}$; then the theta function has the following properties:

- Even

$$
\theta\left(\mathbf{v} ; \omega, \omega^{\prime}\right)=\theta\left(-\mathbf{v} ; \omega, \omega^{\prime}\right)
$$

- Periodic in the real directions $(\omega)$

$\forall \mathbf{n} \in \mathbb{Z}^{2}, \quad \theta\left(\mathbf{v}+\mathbf{n} ; \omega, \omega^{\prime}\right)=\theta\left(\mathbf{v} ; \omega, \omega^{\prime}\right)$

- Quasi-periodic in the complex directions $\left(\omega^{\prime}\right)$

$\forall \mathbf{n} \in \mathbb{Z}^{2}, \quad \theta\left(\mathbf{v}+\left(\omega^{-1} \omega^{\prime}\right) \mathbf{n} ; \omega, \omega^{\prime}\right)=\exp \left(-i \pi \mathbf{n}^{\mathrm{T}}\left(\omega^{-1} \omega^{\prime}\right) \mathbf{n}-2 i \pi \mathbf{v}^{\mathrm{T}} \mathbf{n}\right) \theta\left(\mathbf{v} ; \omega, \omega^{\prime}\right)$.

The zero set of the theta function is of particular importance to the inversion theorem.

Let $\mathbf{K}_{z_{0}}$ be the vector of Riemann constants, with base point $z_{0}$, given by

$$
\begin{aligned}
& \left(\mathbf{K}_{z_{0}}\right)_{1}=\frac{1+B_{11}}{2}-\int_{a_{2}} \mathrm{~d} v_{2}(z) \int_{z_{0}}^{z} \mathrm{~d} v_{1}, \\
& \left(\mathbf{K}_{z_{0}}\right)_{2}=\frac{1+B_{22}}{2}-\int_{a_{1}} \mathrm{~d} v_{1}(z) \int_{z_{0}}^{z} \mathrm{~d} v_{2} .
\end{aligned}
$$

For different base points we get

$$
(2 \omega) \mathbf{K}_{z}=(2 \omega) \mathbf{K}_{z_{0}}+\int_{z_{0}}^{z} \mathrm{~d} \mathbf{u} .
$$


It is known that for $\mathbf{u}=(2 \omega) \mathbf{K}_{z}$ the theta function $\theta\left((2 \omega)^{-1} \mathbf{u} ; \omega, \omega^{\prime}\right)$ vanishes. The set of zeros of $\theta$ is therefore

$$
\Theta=\left\{\mathbf{u} \in \operatorname{Jac}(R): \quad \mathbf{u}=\int_{z_{0}}^{z} \mathrm{~d} \mathbf{u}+2 \omega \mathbf{K}_{z_{0}}, \quad z \in R\right\} .
$$

This set is called the theta divisor; it forms a one dimensional image of $R$ in the two dimensional Jacobi variety, $\operatorname{Jac}(R)$.

Definition 5.3 The fundamental abelian $\sigma$-function (genus 2) is defined as

$$
\sigma\left(\mathbf{u} ; \omega, \omega^{\prime}\right)=\frac{1}{\sqrt[4]{\mathrm{D}(v)}} \mathrm{C} \exp \left(\frac{1}{2} \mathbf{u}^{\mathrm{T}} \eta \omega^{-1} \mathbf{u}\right) \theta\left((2 \omega)^{-1} \mathbf{u} ; \omega, \omega^{\prime}\right),
$$

where

$$
\mathrm{C}=\left(\frac{\pi^{2}}{\operatorname{det}(\omega)}\right)^{\frac{1}{2}}
$$

and $D(v)$ is the discriminant of the curve

$$
\prod_{i=1}^{6}\left(p-p_{i}\right)
$$

For a detailed study of the properties of this function see [4]. It is important to note that:

- it is an entire function on $\operatorname{Jac}(R)$;

- it satisfies the quasi-periodicity properties

$$
\begin{aligned}
\sigma\left(\mathbf{u}+2 \omega \mathbf{n}+2 \omega^{\prime} \mathbf{n}^{\prime} ; \omega, \omega^{\prime}\right)= & \exp \left[2\left(\eta \mathbf{n}+\eta^{\prime} \mathbf{n}^{\prime}\right)^{\mathrm{T}}\left(\mathbf{u}+\omega \mathbf{n}+\omega^{\prime} \mathbf{n}^{\prime}\right)\right] \\
& \times \exp \left[-i \pi \mathbf{n}^{\mathrm{T}} \mathbf{n}^{\prime}\right] \sigma\left(\mathbf{u} ; \omega, \omega^{\prime}\right)
\end{aligned}
$$

and the modular property

$$
\sigma\left(\mathbf{u} ; \tilde{\omega}, \tilde{\omega}^{\prime}\right)=\sigma\left(\mathbf{u} ; \omega, \omega^{\prime}\right),
$$

where

$$
\begin{aligned}
& \tilde{\omega}=\omega d+\omega^{\prime} c, \quad \tilde{\omega}^{\prime}=\omega b+\omega^{\prime} a, \\
& \tilde{\eta}=\eta d+\eta^{\prime} c, \quad \tilde{\eta}^{\prime}=\eta b+\eta^{\prime} a
\end{aligned}
$$

and

$$
\left(\begin{array}{ll}
a^{\mathrm{T}} & b^{\mathrm{T}} \\
c^{\mathrm{T}} & d^{\mathrm{T}}
\end{array}\right) \in S p(4, \mathbb{Z})
$$

$\ddagger$

- $\sigma\left(\mathbf{u} ; \omega, \omega^{\prime}\right)$ is zero when $\mathbf{u} \in \Theta$.

$\ddagger$ The $\sigma$-function is thus independent of the choice of $\mathfrak{a}$ and $\mathfrak{b}$ cycles provided these satisfy $\mathfrak{a}_{i} \circ \mathfrak{b}_{j}=\delta_{i j}$. 
In the following sections we will be concerned with a single Riemann surface, with fixed period matrices $\omega, \omega^{\prime}$, so we will simplify the notation of $\sigma\left(\mathbf{u} ; \omega, \omega^{\prime}\right)$ to $\sigma(\mathbf{u})$. However, when we consider the space and time variation in the original problem, it is important to bear in mind that we are then considering a 2-parameter family of such surfaces with variable period matrices.

As a natural extension of the genus 1 case, we define the logarithmic derivatives of $\sigma(\mathbf{u})$ by

and

$$
\zeta_{i}(\mathbf{u})=\frac{\partial}{\partial u_{i}}[\log \sigma(\mathbf{u})]=\frac{\sigma_{i}}{\sigma}(\mathbf{u}), \quad(i=1,2)
$$

$$
\wp_{i j}(\mathbf{u})=-\frac{\partial^{2}}{\partial u_{i} \partial u_{j}}[\log \sigma(\mathbf{u})]=-\frac{\sigma_{i j}}{\sigma}(\mathbf{u})+\frac{\sigma_{i} \sigma_{j}}{\sigma^{2}}(\mathbf{u}), \quad(i, j=1,2)
$$

where

$$
\sigma_{i}=\frac{\partial \sigma}{\partial u_{i}}, \quad \sigma_{i j}=\frac{\partial^{2} \sigma}{\partial u_{j} \partial u_{i}}, \cdots
$$

The higher order logarithmic derivatives are expressed similarly, for example

$$
\wp_{i j k}(\mathbf{u})=-\frac{\partial^{3}}{\partial u_{i} \partial u_{j} \partial u_{k}}[\log \sigma(\mathbf{u})], \quad(i, j, k=1,2) .
$$

It is well-known that Weierstrass' $\wp$-function satisfies the ODE

$$
\wp^{\prime 2}(x)=4 \wp^{3}(x)-g_{2} \wp(x)-g_{3} .
$$

Similarly, the derivatives of the Kleinian $\wp$-function are related. For genus 2 , the corresponding partial differential equations were established by Baker [1] (quoted in [4]); these are

$$
\begin{aligned}
& \wp_{2222}=6 \wp_{22}^{2}+\frac{1}{2} \mu_{3}+\mu_{4} \wp_{22}+4 \wp_{12}, \\
& \wp_{2221}=6 \wp_{22} \wp_{12}+\mu_{4} \wp_{12}-2 \wp_{11}, \\
& \wp_{2211}=2 \wp_{22} \wp_{11}+4 \wp_{12}^{2}+\frac{1}{2} \mu_{3} \wp_{12}, \\
& \wp_{2111}=6 \wp_{12} \wp_{11}+\mu_{2} \wp_{12}-\frac{1}{2} \mu_{1} \wp_{22}-\mu_{0}, \\
& \wp_{1111}=6 \wp_{11}^{2}-3 \mu_{0} \wp_{22}+\mu_{1} \wp_{12}+\mu_{2} \wp_{11}-\frac{1}{2} \mu_{0} \mu_{4}+\frac{1}{8} \mu_{1} \mu_{3} .
\end{aligned}
$$

Further, the Baker addition formula for genus 2 is given by

$$
\frac{\sigma(\mathbf{u}+\mathbf{v}) \sigma(\mathbf{u}-\mathbf{v})}{\sigma(\mathbf{u})^{2} \sigma(\mathbf{v})^{2}}=\wp_{22}(\mathbf{u}) \wp_{12}(\mathbf{v})-\wp_{12}(\mathbf{u}) \wp_{22}(\mathbf{v})+\wp_{11}(\mathbf{v})-\wp_{11}(\mathbf{u})
$$

for $\mathbf{u}, \mathbf{v} \notin \Theta .[4]$

\subsection{Jacobi's inversion theorem}

We can now use the above setting to state the inversion theorem [4]. 
Theorem 5.1 Let $V_{2}$ be a hyperelliptic curve of genus 2 defined by

$$
y^{2}=\sum_{i=0}^{6} \rho_{i} x^{i}
$$

with branch points $p_{i}$ as in figure 8. Let

$$
u_{i}=\int_{p_{2}}^{x_{a}} \frac{x^{i-1}}{y} \mathrm{~d} x+\int_{p_{4}}^{x_{b}} \frac{x^{i-1}}{y} \mathrm{~d} x
$$

where $\left(y_{a}, x_{a}\right) \neq\left(-y_{b}, x_{b}\right)$.

The Abel preimage of the point $\mathbf{u} \in \operatorname{Jac}\left(V_{2}\right)$ is then given by the set $\left\{\left(y_{a}, x_{a}\right),\left(y_{b}, x_{b}\right)\right\} \in$ $\left(V_{2}\right)^{2}$, where $\left\{x_{a}, x_{b}\right\}$ are the zeros of the polynomial

$$
\mathcal{P}(x ; \mathbf{u})=x^{2}-\wp_{22}(\mathbf{u}) x-\wp_{12}(\mathbf{u})
$$

and $\left\{y_{a}, y_{b}\right\}$ are given by

$$
y_{k}=-\left.\frac{\partial \mathcal{P}(x ; \mathbf{u})}{\partial u_{g}}\right|_{x=x_{k}} .
$$

We note that the Jacobi inversion theorem can not be applied to our problem direcly, as we are concerned with a second kind differential, and a divisor of degree 1 , not 2 . However, by restricting the values of $\mathbf{u}$ to the theta divisor, which is an image of the curve

$$
s^{2}=4 \prod_{i=1}^{5}\left(t-t_{i}\right)
$$

we can use the method given in [6] to invert integrals like

$$
\mathbf{u}\left(z_{1}\right)=\int_{\infty}^{z_{1}} \mathrm{~d} \mathbf{u}, \quad \mathbf{u}\left(z_{1}\right) \in \Theta
$$

Note that the Jacobi inversion formula can not be used here since if $\mathbf{u}$ is a zero of the $\theta$-function, then it is also a zero of the $\sigma$-function, so that the quadratic equation

$$
\mathcal{P}(t ; \mathbf{u})=t^{2}-\wp_{22}(\mathbf{u}) t-\wp_{12}(\mathbf{u})=0
$$

is singular and its roots are undefined. This problem is overcome by noting that if $t_{a}$ and $t_{b}$ are the two solutions of $\mathcal{P}(t ; \mathbf{u})=0$, then

$$
t_{a}=\lim _{t_{b} \rightarrow \infty} \frac{t_{a} t_{b}}{t_{a}+t_{b}}
$$

We can thus define the inverse of (32) by

$$
\begin{aligned}
z_{1}=t_{a}=\lim _{t_{b} \rightarrow \infty} \frac{t_{a} t_{b}}{t_{a}+t_{b}} & =\lim _{\sigma \rightarrow 0}\left(-\frac{\wp_{12}(\mathbf{u})}{\wp_{22}(\mathbf{u})}\right) \\
& =\lim _{\sigma \rightarrow 0}\left(\frac{\sigma \sigma_{12}-\sigma_{1} \sigma_{2}}{\sigma_{2}^{2}-\sigma \sigma_{22}}\right)=-\frac{\sigma_{1}}{\sigma_{2}}(\mathbf{u})
\end{aligned}
$$

where $\mathbf{u} \in \Theta$ since

$$
\begin{aligned}
\lim _{t_{b} \rightarrow \infty} \mathbf{u} & =\lim _{t_{b} \rightarrow \infty}\left(\int_{\infty}^{t_{a}} \mathrm{~d} \mathbf{u}+\int_{\infty}^{t_{b}} \mathrm{~d} \mathbf{u}\right) \\
& =\int_{\infty}^{t_{a}} \mathrm{~d} \mathbf{u} .
\end{aligned}
$$




\section{Evaluation of the integral}

The integrand (27) can now be transformed using the substitution (33). In this case we define

$$
\begin{aligned}
\mathrm{d} u_{1} & =\frac{1}{s} \mathrm{~d} t \\
\mathrm{~d} u_{2} & =\frac{t}{s} \mathrm{~d} t
\end{aligned}
$$

where $s$ is as given in (26), and let

$$
u_{i}(t)=\int_{\infty}^{t} \mathrm{~d} u_{i} \quad(i=1,2) .
$$

Lemma 6.1 Let $t=-\left(\sigma_{1} / \sigma_{2}\right)(\mathbf{u})$ and let $d u_{1}, d u_{2}$ be defined by (33) and (34) respectively. Then for $\mathbf{u} \in \Theta$ the integrand $\varphi(p) \mathrm{d} p$ given by (25) may be rewritten

$$
\varphi(p) \mathrm{d} p=k\left[\mathbf{A}^{\mathrm{T}} \cdot \mathrm{d} \mathbf{u}-c\left(\frac{\sigma_{2}}{\sigma_{1}}(\mathbf{u}) \mathrm{d} u_{1}\right)-\left(\frac{\sigma_{2}^{2}}{\sigma_{1}^{2}}(\mathbf{u}) \mathrm{d} u_{1}\right)\right]
$$

where $\mathbf{A}^{\mathrm{T}}=(b, a)$.

In section 3 we specified that as $p \rightarrow \infty$, on either sheet of the Riemann surface, the residue of the function $\varphi(p)$ must be zero. It therefore follows that

$$
\psi(\mathbf{u})=\frac{\sigma_{2}^{2}}{\sigma_{1}^{2}}(\mathbf{u})+c \frac{\sigma_{2}}{\sigma_{1}}(\mathbf{u})
$$

must have two double poles with zero residue as $\sigma_{1} \rightarrow 0$ on the divisor. We will now verify this by calculating the associated Taylor series.

Let $\pm \mathbf{u}_{0}$, where $\mathbf{u}_{0}=\left(u_{0,1}, u_{0,2}\right)$, denote the poles of $\psi(\mathbf{u})$. The corresponding points in the $t$ and $p$ variables are given in Table 1.

Table 1. A list of branch points $\left(p_{i}\right)$ and poles $\left(\infty_{ \pm}\right)$of $\lambda(p)$ with the corresponding points in the $t$ and $\mathbf{u}$ variables.

\begin{tabular}{llllllll}
\hline$(p)$ & $p_{1}$ & $p_{2}$ & $p_{3}$ & $p_{4}$ & $p_{5}$ & $p_{6}$ & $\infty_{ \pm}$ \\
\hline$(t)$ & $t_{1}$ & $t_{2}$ & $t_{3}$ & $t_{4}$ & $t_{5}$ & $\infty$ & $0_{ \pm}$ \\
$(\mathbf{u})$ & $\mathbf{u}_{1}$ & $\mathbf{u}_{2}$ & $\mathbf{u}_{3}$ & $\mathbf{u}_{4}$ & $\mathbf{u}_{5}$ & $\mathbf{0}$ & $\pm \mathbf{u}_{0}$ \\
\hline
\end{tabular}

If we expand the two terms in $\psi(\mathbf{u})$ about $\mathbf{u}_{0}$, then we have

$$
\begin{aligned}
\frac{\sigma_{2}^{2}}{\sigma_{1}^{2}}(\mathbf{u}) & =\frac{\sigma_{2}^{2}}{\sigma_{1}^{2}}\left(\mathbf{u}_{0}+\left(\mathbf{u}-\mathbf{u}_{0}\right)\right) \\
& =\frac{\sigma_{2}^{2}+2 \sigma_{2} \sigma_{12}\left(u_{1}-u_{0,1}\right)+2 \sigma_{2} \sigma_{22}\left(u_{2}-u_{0,2}\right)+\cdots}{\sigma_{11}^{2}\left(u_{1}-u_{0,1}\right)^{2}+2 \sigma_{11} \sigma_{12}\left(u_{1}-u_{0,1}\right)\left(u_{2}-u_{0,2}\right)+\cdots}
\end{aligned}
$$

and

$$
\begin{aligned}
\frac{\sigma_{2}}{\sigma_{1}}(\mathbf{u}) & =\frac{\sigma_{2}}{\sigma_{1}}\left(\mathbf{u}_{0}+\left(\mathbf{u}-\mathbf{u}_{0}\right)\right) \\
& =\frac{\sigma_{2}+\sigma_{12}\left(u_{1}-u_{0,1}\right)+\sigma_{22}\left(u_{2}-u_{0,2}\right)+\cdots}{\sigma_{11}\left(u_{1}-u_{0,1}\right)+\sigma_{12}\left(u_{2}-u_{0,2}\right)+\cdots}
\end{aligned}
$$


where each of the $\sigma$-function derivatives on the RHS is evaluated at $\mathbf{u}_{0}$. Expanding these further we obtain a Taylor series in $\left(u_{1}-u_{0,1}\right)$ and $\left(u_{2}-u_{0,2}\right)$. This may be simplified to a series in one variable as follows. From the definition of $u_{i}(28)$ and the fact $t=-\frac{\sigma_{1}}{\sigma_{2}}(\mathbf{u})$, we have

$$
\left(u_{i}-u_{0, i}\right)=\int_{0}^{t} \mathrm{~d} u_{i} \quad(i=1,2) .
$$

Now, as $\mathbf{u}_{0}$ is a regular point on the hyperelliptic curve, we can expand these integrals in terms of the local parameter $t$. We thus find that $\S$

$$
\begin{aligned}
& \left(u_{1}-u_{0,1}\right)=\frac{1}{\sqrt{\mu_{0}}} t-\frac{1}{4} \frac{\mu_{1}}{\mu_{0}^{3 / 2}} t^{2}+O\left(t^{3}\right), \\
& \left(u_{2}-u_{0,2}\right)=\frac{1}{2} \frac{1}{\sqrt{\mu_{0}}} t^{2}-\frac{1}{6} \frac{\mu_{1}}{\mu_{0}^{3 / 2}} t^{3}+O\left(t^{4}\right)
\end{aligned}
$$

and so we can write $\left(u_{2}-u_{0,2}\right)$ in terms of $\left(u_{1}-u_{0,1}\right)$ as follows

$$
\begin{aligned}
\left(u_{2}-u_{0,2}\right)= & \frac{1}{2} \sqrt{\mu_{0}}\left(u_{1}-u_{0,1}\right)^{2}+\frac{1}{12} \mu_{1}\left(u_{1}-u_{0,1}\right)^{3} \\
& +O\left(\left(u_{1}-u_{0,1}\right)^{4}\right) .
\end{aligned}
$$

Substituting (37) and (37) into the Taylor expansion of $\psi(\mathbf{u})$ we have

$$
\begin{aligned}
\psi(\mathbf{u})= & \psi\left(\mathbf{u}_{0}+\left(\mathbf{u}-\mathbf{u}_{\mathbf{0}}\right)\right) \\
= & \left(\frac{\sigma_{2}^{2}}{\sigma_{11}^{2}}\right) \frac{1}{\left(u_{1}-u_{0,1}\right)^{2}} \\
& +\left(2 \frac{\sigma_{2} \sigma_{12}}{\sigma_{11}{ }^{2}}+c \frac{\sigma_{2}}{\sigma_{11}}-\sqrt{\mu_{0}} \frac{\sigma_{2}^{2} \sigma_{12}}{\sigma_{11}{ }^{3}}-\frac{\sigma_{2}^{2} \sigma_{111}}{\sigma_{11}{ }^{3}}\right) \frac{1}{\left(u_{1}-u_{0,1}\right)}+O(1) .
\end{aligned}
$$

This expression may be simplified further using the relations between the derivatives of the $\sigma$-function on the divisor and at the point $\mathbf{u}_{0}$. These can be calculated by comparing the coefficients in the expansions of the five partial differential equations (31) - (31) and the identity

$$
\sigma(\mathbf{u})=0 \quad \text { for } \mathbf{u} \in \Theta .
$$

This method and the full set of relations holding on $\Theta$ for the third order derivatives are given in Appendix A and Appendix B.

The case of $\sigma_{11}\left(\mathbf{u}_{0}\right)$, however, is slightly different. For a general point on the divisor the expression for $\sigma_{11}(\mathbf{u})$ is as given in (A.1), but here a direct substition of $\mathbf{u}=\mathbf{u}_{0}$ leads to a sign ambiguity. This is resolved by evaluating $\sigma_{11}\left(\mathbf{u}_{0}\right)$ from the Taylor series of $\sigma(\mathbf{u})$ about $\mathbf{u}_{0}$. Expanding along the divisor, we have

$$
\begin{aligned}
0 & =\sigma(\mathbf{u}) \\
& =\sigma\left(\mathbf{u}_{0}+\left(\mathbf{u}-\mathbf{u}_{0}\right)\right)
\end{aligned}
$$

$\S$ In practice, these series were evaluated to high order using Maple (version 6). 


$$
=\sigma_{2}\left(u_{2}-u_{0,2}\right)+\frac{1}{2} \sigma_{11}\left(u_{1}-u_{0,1}\right)^{2}+\cdots
$$

(since both $\sigma$ and $\sigma_{1}$ are zero)

$$
=\left(\frac{1}{2} \sigma_{2} \sqrt{\mu_{0}}+\frac{1}{2} \sigma_{11}\right)\left(u_{1}-u_{0,1}\right)^{2}+O\left(\left(u_{1}-u_{0,1}\right)^{3}\right)
$$

(from (37)).

We therefore have

$$
\sigma_{11}\left(\mathbf{u}_{0}\right)=-\sqrt{\mu_{0}} \sigma_{2}\left(\mathbf{u}_{0}\right) .
$$

We can now use (B.1) and (37) to evaluate $\psi(\mathbf{u})$ :

$$
\begin{aligned}
\psi(\mathbf{u})= & \psi\left(\mathbf{u}_{0}+\left(\mathbf{u}-\mathbf{u}_{0}\right)\right) \\
= & \left(\frac{1}{\mu_{0}}\right) \frac{1}{\left(u_{1}-u_{0,1}\right)^{2}} \\
& +\left(-c \frac{1}{\sqrt{\mu_{0}}}-\frac{1}{2} \frac{\mu_{1}}{\mu_{0}^{(3 / 2)}}\right) \frac{1}{\left(u_{1}-u_{0,1}\right)}+O(1) ;
\end{aligned}
$$

so for the residue to be zero we require that

$$
c=-\frac{1}{2} \frac{\mu_{1}}{\mu_{0}} .
$$

From equation (25) and identity (24) we have

$$
\begin{aligned}
c & =\sum_{i=1}^{3}\left(p_{6}-\hat{p}_{i}\right)=3 p_{6}-\sum_{i=1}^{3} \hat{p}_{i} \\
& =3 p_{6}-\frac{1}{2} \sum_{i=1}^{6} p_{i}=\frac{1}{2} \sum_{i=1}^{5}\left(p_{6}-p_{i}\right)
\end{aligned}
$$

and from the definitions of $\mu_{0}$ and $\mu_{1}$ in equation (25) we have that

$$
\begin{aligned}
-\frac{1}{2} \frac{\mu_{1}}{\mu_{0}} & =-\frac{1}{2}\left(4 \frac{5 p_{6}-p_{5}-p_{4}-p_{3}-p_{2}-p_{1}}{\prod_{i=1}^{5}\left(p_{6}-p_{i}\right)}\right)\left(-\frac{1}{4} \prod_{i=1}^{5}\left(p_{6}-p_{i}\right)\right) \\
& =\frac{1}{2} \sum_{i=1}^{5}\left(p_{6}-p_{i}\right) .
\end{aligned}
$$

Thus $\psi(\mathbf{u})$ is of the correct form.

We now consider the function

$$
\Phi(\mathbf{u})=-\frac{1}{\mu_{0}} \frac{\sigma_{11}}{\sigma_{1}}(\mathbf{u})
$$

Its derivative along the divisor with respect to $u_{1}$ is

$$
\begin{aligned}
\frac{\mathrm{d}}{\mathrm{d} u_{1}} \Phi(\mathbf{u}) & =\frac{\partial}{\partial u_{1}} \Phi(\mathbf{u})-\left(\frac{\sigma_{1}}{\sigma_{2}}(\mathbf{u})\right) \frac{\partial}{\partial u_{2}} \Phi(\mathbf{u}) \\
& =-\frac{1}{\mu_{0}}\left[\frac{\sigma_{111}}{\sigma_{1}}-\frac{\sigma_{11}^{2}}{\sigma_{1}^{2}}-\left(\frac{\sigma_{1}}{\sigma_{2}}\right)\left(\frac{\sigma_{112}}{\sigma_{1}}-\frac{\sigma_{11} \sigma_{12}}{\sigma_{1}^{2}}\right)\right] \\
& =\frac{1}{\mu_{0}}\left[\frac{\sigma_{111}}{\sigma_{1}}-\frac{\sigma_{11}^{2}}{\sigma_{1}^{2}}-\frac{\sigma_{112}}{\sigma_{2}}+\frac{\sigma_{11} \sigma_{12}}{\sigma_{1} \sigma_{2}}\right] .
\end{aligned}
$$


Substituting identities (A.1), (A.1) and (A.1) into this gives

$$
\begin{aligned}
\frac{\mathrm{d}}{\mathrm{d} u_{1}} \Phi(\mathbf{u}) & =\frac{\sigma_{2}^{2}}{\sigma_{1}^{2}}-\frac{1}{2} \frac{\mu_{1}}{\mu_{0}} \frac{\sigma_{2}}{\sigma_{1}} \\
& =\psi(\mathbf{u}) .
\end{aligned}
$$

The function $\Phi(\mathbf{u})$ may be written in terms of $\zeta_{1}$-functions using the following addition formulae.

Theorem 6.1 [6] Let $\mathbf{u}, \mathbf{v} \in \Theta$, then the following addition formula holds

$$
\begin{aligned}
& \zeta_{1}(\mathbf{u}+\mathbf{v})+\zeta_{1}(\mathbf{u}-\mathbf{v})= \\
& 2 \frac{\sigma_{2}(\mathbf{v}) \sigma_{1}(\mathbf{u}) \sigma_{12}(\mathbf{u})}{\sigma_{2}(\mathbf{u})\left(\sigma_{2}(\mathbf{u}) \sigma_{1}(\mathbf{v})-\sigma_{1}(\mathbf{u}) \sigma_{2}(\mathbf{v})\right)}-\frac{\sigma_{2}(\mathbf{v}) \sigma_{11}(\mathbf{u})}{\sigma_{2}(\mathbf{u}) \sigma_{1}(\mathbf{v})-\sigma_{1}(\mathbf{u}) \sigma_{2}(\mathbf{v})} \\
& -\frac{\sigma_{2}(\mathbf{v}) \sigma_{1}(\mathbf{u})^{2} \sigma_{22}(\mathbf{u})}{\sigma_{2}(\mathbf{u})^{2}\left(\sigma_{2}(\mathbf{u}) \sigma_{1}(\mathbf{v})-\sigma_{1}(\mathbf{u}) \sigma_{2}(\mathbf{v})\right)}+2 \frac{\sigma_{12}(\mathbf{u})}{\sigma_{2}(\mathbf{u})}-\frac{\sigma_{22}(\mathbf{u}) \sigma_{1}(\mathbf{u})}{\sigma_{2}(\mathbf{u})^{2}}
\end{aligned}
$$

Proof 6.1 The partial derivative with respect to $u_{1}$ of the logarithm of the Baker addition formula (31) is

$$
\begin{aligned}
L H S & =\frac{\partial}{\partial u_{1}}\left[\ln \left(\frac{\sigma(\mathbf{u}+\mathbf{v}) \sigma(\mathbf{u}-\mathbf{v})}{\sigma(\mathbf{u})^{2} \sigma(\mathbf{v})^{2}}\right)\right] \\
& =\frac{\sigma_{1}(\mathbf{u}+\mathbf{v})}{\sigma(\mathbf{u}+\mathbf{v})}+\frac{\sigma_{1}(\mathbf{u}-\mathbf{v})}{\sigma(\mathbf{u}-\mathbf{v})}-2 \frac{\sigma_{1}(\mathbf{u})}{\sigma(\mathbf{u})} \\
& =\zeta_{1}(\mathbf{u}+\mathbf{v})+\zeta_{1}(\mathbf{u}-\mathbf{v})-2 \zeta_{1}(\mathbf{u}) ; \\
R H S & =\frac{\partial}{\partial u_{1}} \ln \left[\wp_{22}(\mathbf{u}) \wp_{12}(\mathbf{v})-\wp_{12}(\mathbf{u}) \wp_{22}(\mathbf{v})+\wp_{11}(\mathbf{v})-\wp_{11}(\mathbf{u})\right] \\
& =\frac{\wp_{122}(\mathbf{u}) \wp_{12}(\mathbf{v})-\wp_{112}(\mathbf{u}) \wp_{22}(\mathbf{v})-\wp_{111}(\mathbf{u})}{\wp_{22}(\mathbf{u}) \wp_{12}(\mathbf{v})-\wp_{12}(\mathbf{u}) \wp_{22}(\mathbf{v})+\wp_{11}(\mathbf{v})-\wp_{11}(\mathbf{u})} .
\end{aligned}
$$

If we now add $2 \zeta_{1}(\mathbf{u})$ to both sides of this equation, we have

$\zeta_{1}(\mathbf{u}+\mathbf{v})+\zeta_{1}(\mathbf{u}-\mathbf{v})=\frac{\wp_{122}(\mathbf{u}) \wp_{12}(\mathbf{v})-\wp_{112}(\mathbf{u}) \wp_{22}(\mathbf{v})-\wp_{111}(\mathbf{u})}{\wp_{22}(\mathbf{u}) \wp_{12}(\mathbf{v})-\wp_{12}(\mathbf{u}) \wp_{22}(\mathbf{v})+\wp_{11}(\mathbf{v})-\wp_{11}(\mathbf{u})}+2 \zeta_{1}(\mathbf{u})$.

Expanding the RHS of (39) in terms of $\mathbf{u} \in \Theta$, and substituting in the relations for the $\sigma$ derivatives, gives a series in $\xi$ with first term of order 1 . II If we then repeat this for $\mathbf{v} \in \Theta$ and let $\xi \rightarrow 0$, we obtain the required result.

Corollary 6.1 Let $\mathbf{u}, \mathbf{u}_{\mathbf{0}} \in \Theta$ and let $\sigma_{1}\left(\mathbf{u}_{\mathbf{0}}\right)=0$, then

$$
\begin{aligned}
\zeta_{1}\left(\mathbf{u}+\mathbf{u}_{\mathbf{0}}\right)+\zeta_{1}\left(\mathbf{u}-\mathbf{u}_{\mathbf{0}}\right) & =\frac{\sigma_{11}}{\sigma_{1}}(\mathbf{u}), \\
\zeta_{1}\left(\mathbf{u}+\mathbf{u}_{\mathbf{0}}\right)-\zeta_{1}\left(\mathbf{u}-\mathbf{u}_{\mathbf{0}}\right) & =\sqrt{\mu_{0}} \frac{\sigma_{2}}{\sigma_{1}}(\mathbf{u})+2 \frac{\sigma_{12}}{\sigma_{2}}\left(\mathbf{u}_{\mathbf{0}}\right) .
\end{aligned}
$$

Proof 6.2 (a) Set $\mathbf{v}=\mathbf{u}_{\mathbf{0}}$ in addition theorem 6.1 and use the identity $\sigma_{1}\left(\mathbf{u}_{0}\right)=0$.

(b) Similarly, set $\mathbf{u}=\mathbf{u}_{\mathbf{0}}$ in theorem 6.1.

\| The Taylor series were calculated using Maple (version 6). 
Thus, for $\mathbf{u} \in \Theta$ we have

$$
\Phi(\mathbf{u})=-\frac{1}{\mu_{0}} \frac{\sigma_{11}}{\sigma_{1}}(\mathbf{u})=-\frac{1}{\mu_{0}}\left[\zeta_{1}\left(\mathbf{u}+\mathbf{u}_{0}\right)+\zeta_{1}\left(\mathbf{u}-\mathbf{u}_{0}\right)\right] .
$$

The differential $\varphi(p) \mathrm{d} p$ given in lemma 6.1 is therefore expressible as the derivative of known functions:

$$
\varphi(p) \mathrm{d} p=k\left[\mathbf{A}^{\mathrm{T}} \cdot \mathrm{d} \mathbf{u}-\left(\frac{\mathrm{d}}{\mathrm{d} u_{1}} \Phi(\mathbf{u})\right) \mathrm{d} u_{1}\right]
$$

where

$$
k= \pm \sqrt{\mu_{0}}= \pm\left(\frac{-4}{\prod_{i=1}^{5}\left(p_{6}-p_{i}\right)}\right)^{\frac{1}{2}}
$$

Hence, substituting

$$
p=p_{6}+\frac{\sigma_{2}}{\sigma_{1}}(\mathbf{u})
$$

into (22) we have

$$
\begin{aligned}
\lambda(p) & =p+\int_{\infty}^{p}\left[\varphi\left(p^{\prime}\right)-1\right] \mathrm{d} p \\
& =\left(p_{6}+\frac{\sigma_{2}}{\sigma_{1}}(\mathbf{u})\right)+\int_{0}^{\frac{1}{p_{6}-p}}\left[k \mathbf{A}^{\mathrm{T}} \cdot \mathrm{d} \mathbf{u}-k\left(\frac{\mathrm{d}}{\mathrm{d} u_{1}} \Phi(\mathbf{u})\right) \mathrm{d} u_{1}-\frac{\mathrm{d} t}{t^{2}}\right] \\
& =\left(p_{6}+\frac{\sigma_{2}}{\sigma_{1}}(\mathbf{u})\right)+\left\{k \mathbf{A}^{\mathrm{T}} \cdot \mathbf{u}+\frac{1}{k}\left[\zeta_{1}\left(\mathbf{u}+\mathbf{u}_{0}\right)+\zeta_{1}\left(\mathbf{u}-\mathbf{u}_{0}\right)\right]-\frac{\sigma_{2}}{\sigma_{1}}(\mathbf{u})\right\}+\widetilde{C}
\end{aligned}
$$

The constant $\widetilde{C}$ can be found by evaluating both sides at any convenient point. For example, if we set $p=p_{6} \Leftrightarrow \mathbf{u}=\mathbf{0}$, then

$$
\widetilde{C}=\lambda\left(p_{6}\right)-p_{6}=\lambda_{3}^{0}-p_{6} .
$$

Alternatively, setting $p \rightarrow \infty_{+} \Leftrightarrow \mathbf{u} \rightarrow+\mathbf{u}_{0}$, we find

$$
\lambda(p)-p=k \mathbf{A}^{\mathrm{T}} \cdot \mathbf{u}+\frac{1}{k}\left[\zeta_{1}\left(\mathbf{u}+\mathbf{u}_{0}\right)+\zeta_{1}\left(\mathbf{u}-\mathbf{u}_{0}\right)\right]-\frac{\sigma_{2}}{\sigma_{1}}(\mathbf{u})+\widetilde{C} \rightarrow 0 .
$$

Hence

$$
\widetilde{C}=-k\left(\mathbf{A}^{\mathrm{T}} \cdot \mathbf{u}_{0}\right)-\frac{1}{k} \zeta_{1}\left(2 \mathbf{u}_{0}\right)-\lim _{\mathbf{u} \rightarrow \mathbf{u}_{0}}\left[\frac{1}{k} \zeta_{1}\left(\mathbf{u}-\mathbf{u}_{0}\right)-\frac{\sigma_{2}}{\sigma_{1}}(\mathbf{u})\right] .
$$

Expanding this near $\mathbf{u}_{0}$ we have

$$
\begin{aligned}
-\frac{1}{k} \zeta_{1}\left(\mathbf{u}-\mathbf{u}_{0}\right)+\frac{\sigma_{2}}{\sigma_{1}}\left(\mathbf{u}_{0}+\left(\mathbf{u}-\mathbf{u}_{0}\right)\right)= & \lim _{u_{1} \rightarrow u_{0,1}}\left[\left(-\frac{1}{k}\left(u_{1}-u_{0,1}\right)^{-1}+O\left(\left(u_{1}-u_{0,1}\right)\right)\right)\right. \\
& \left.+\left(-\frac{1}{\sqrt{\mu_{0}}}\left(u_{1}-u_{0,1}\right)^{-1}+\left(\frac{1}{4} \frac{\mu_{1}}{\mu_{0}}\right)+O\left(\left(u_{1}-u_{0,1}\right)\right)\right)\right] \\
= & \frac{1}{4} \frac{\mu_{1}}{\mu_{0}}
\end{aligned}
$$

(setting $\left.k=-\sqrt{\mu_{0}}\right)$. 
It follows that

$$
\begin{aligned}
\widetilde{C} & =\sqrt{\mu_{0}} \mathbf{A}^{\mathrm{T}} \cdot \mathbf{u}_{0}+\frac{1}{\sqrt{\mu_{0}}} \zeta_{1}\left(2 \mathbf{u}_{0}\right)+\frac{1}{4} \frac{\mu_{1}}{\mu_{0}} \\
& =\sqrt{\mu_{0}} \mathbf{A}^{\mathrm{T}} \cdot \mathbf{u}_{0}
\end{aligned}
$$

(using identity (C.1) in appendix Appendix C).

Hence, analogously to (21), we obtain the following result.

\section{Theorem 6.2 Let}

and

$$
\begin{aligned}
\lambda(p) & =p+\int_{\infty}^{p} \frac{\prod_{i=1}^{3}\left(p^{\prime}-\hat{p}_{i}\right)}{\sqrt{\prod_{i=1}^{6}\left(p^{\prime}-p_{i}\right)}} \mathrm{d} p^{\prime} \\
k & =-\left(\frac{-4}{\prod_{i=1}^{5}\left(p_{6}-p_{i}\right)}\right)^{\frac{1}{2}}
\end{aligned}
$$

$$
\mathbf{A}^{\mathrm{T}}=\left(\sum_{i=1}^{3} \prod_{j>i}^{3}\left(p_{6}-\hat{p}_{i}\right)\left(p_{6}-\hat{p}_{j}\right), \prod_{i=1}^{3}\left(p_{6}-\hat{p}_{i}\right)\right) .
$$

Then if we set

$$
p=p_{6}+\frac{\sigma_{2}}{\sigma_{1}}(\mathbf{u})
$$

with $\mathbf{u}, \mathbf{u}_{0} \in \Theta$ and $\sigma_{1}\left(\mathbf{u}_{0}\right)=0$, we have

$$
\begin{aligned}
\lambda(p) & =p_{6}+\frac{1}{k}\left[\zeta_{1}\left(\mathbf{u}+\mathbf{u}_{0}\right)+\zeta_{1}\left(\mathbf{u}-\mathbf{u}_{0}\right)\right]+k \mathbf{A}^{\mathrm{T}} \cdot\left(\mathbf{u}-\mathbf{u}_{0}\right) \\
& =p+\frac{1}{k}\left[\zeta_{1}\left(\mathbf{u}+\mathbf{u}_{0}\right)+\zeta_{1}\left(\mathbf{u}-\mathbf{u}_{0}\right)\right]-\frac{\sigma_{2}}{\sigma_{1}}(\mathbf{u})+k \mathbf{A}^{\mathrm{T}} \cdot\left(\mathbf{u}-\mathbf{u}_{0}\right)
\end{aligned}
$$

on sheet $R_{2}^{+}$of the Riemann surface

$$
R_{2}=\left\{(v, p) \in \mathbb{C}^{2}: v^{2}=\prod_{i=1}^{6}\left(p-p_{i}\right)\right\}
$$

associated with the relation $p \rightarrow \infty_{+} \Leftrightarrow \mathbf{u} \rightarrow \mathbf{u}_{0}$.

\section{Summary}

In the genus 2 case the conformal map from $\Gamma_{1}$ to $\Gamma_{2}, p \rightarrow \lambda(p)$, is described as follows. We fix three real parameters $\lambda_{n}^{0}(n=1,2,3)$ and require that the three variables $\hat{\lambda}_{n}$ satisfy

$$
\operatorname{Re}\left(\hat{\lambda}_{n}\right)=\lambda_{n}^{0}
$$

The mapping $\lambda(p)$ is then given by the Schwarz-Christoffel integral

$$
\lambda(p)=p+\int_{\infty}^{p}\left[\frac{\prod_{i=1}^{3}\left(p^{\prime}-\hat{p}_{i}\right)}{\sqrt{\prod_{i=1}^{6}\left(p^{\prime}-p_{i}\right)}}-1\right] \mathrm{d} p^{\prime} .
$$


Here the nine parameters $p_{i}(i=1,2, \ldots, 6), \hat{p}_{j}(j=1,2,3)$ are fixed by the nine relations

$$
\begin{array}{lc}
\int_{\infty}^{p_{2 n-1}}[\varphi(p)-1] \mathrm{d} p+p_{2 n-1}=\lambda_{n}^{0} & (n=1,2,3) \\
\int_{p_{2 n-1}}^{p_{2 n}} \varphi(p) \mathrm{d} p=0 & (n=1,2,3)
\end{array}
$$

and

$$
\int_{p_{2 n-1}}^{\hat{p}_{n}} \varphi(p) \mathrm{d} p=i \operatorname{Im}\left(\hat{\lambda}_{n}\right) \quad(n=1,2,3) .
$$

The explicit form of $\lambda(p)$, depending on the 3 parameters $\operatorname{Im}\left(\hat{\lambda}_{n}\right)$, is given by

$$
\lambda(p)=p+\frac{1}{k}\left[\zeta_{1}\left(\mathbf{u}+\mathbf{u}_{0}\right)+\zeta_{1}\left(\mathbf{u}-\mathbf{u}_{0}\right)\right]-\frac{\sigma_{2}}{\sigma_{1}}(\mathbf{u})+k \mathbf{A}^{\mathrm{T}} \cdot\left(\mathbf{u}-\mathbf{u}_{0}\right)
$$

with

$$
p=p_{6}+\frac{\sigma_{2}}{\sigma_{1}}(\mathbf{u})
$$

and $\sigma(\mathbf{u})=0$. This is a highly implicit and transcendental expression.

Its asymptotics are given as follows

$$
\lambda(p)=p+\frac{A_{0}}{p}+O\left(\frac{1}{p^{2}}\right)
$$

where

$$
A_{0}=-\frac{1}{8} \frac{\mu_{1}^{2}}{\mu_{0}^{2}}+\frac{1}{2} \frac{\mu_{2}}{\mu_{0}}+\sum_{i=1}^{3} \prod_{j>i}^{3}\left(p_{6}-\hat{p}_{i}\right)\left(p_{6}-\hat{p}_{j}\right) .
$$

Similar expressions may be found in principle for higher moments $A_{n}$.

If $\lambda(p)$ is required to satisfy Benney's equation (10) and to be of this form, then the $\hat{\lambda}_{n}$ satisfy

$$
\frac{\partial \hat{\lambda}_{n}}{\partial t}+\hat{p}_{n} \frac{\partial \hat{\lambda}_{n}}{\partial x}=0 \quad(n=1,2,3)
$$

Further, the $\hat{p}_{n}$ and $A_{0}$ must satisfy the Gibbons-Tsarev equations [7], [9]:

$$
\begin{aligned}
\frac{\partial \hat{p}_{j}}{\partial \hat{\lambda}_{i}} & =\left(\frac{\partial A_{0}}{\partial \hat{\lambda}_{i}}\right) \frac{1}{\hat{p}_{i}-\hat{p}_{j}}, \\
\frac{\partial^{2} A_{0}}{\partial \hat{\lambda}_{i} \partial \hat{\lambda}_{j}} & =2\left(\frac{\partial A_{0}}{\partial \hat{\lambda}_{i}} \frac{\partial A_{0}}{\partial \hat{\lambda}_{j}}\right) \frac{1}{\left(\hat{p}_{i}-\hat{p}_{j}\right)^{2}} .
\end{aligned}
$$

It would be interesting to find a more explicit form of this solution.

\section{Appendix A. Differential relations holding on the divisor}

The differential relations for derivatives of $\sigma(\mathbf{u}), \mathbf{u} \in \Theta$, are evaluated as follows.

For a general point $\tilde{\mathbf{u}} \in \Theta$ we have

$$
\left(u_{i}-\tilde{u}_{i}\right)=\int_{\infty}^{t} \mathrm{~d} u_{i} \quad(i=1,2) .
$$


Setting $t=\xi^{-2}$ and expanding the RHS in terms of this local parameter, $\xi$, we obtain the series

and

$$
\left(u_{1}-\tilde{u}_{1}\right)=-\frac{1}{3} \xi^{3}+\frac{\mu_{4}}{40} \xi^{5}+O\left(\xi^{7}\right)
$$

$$
\left(u_{2}-\tilde{u}_{2}\right)=-\xi+\frac{\mu_{4}}{24} \xi^{3}+O\left(\xi^{5}\right)
$$

If we now, for example, take the first equation (31)

$$
\wp_{2222}-6 \wp_{22}^{2}-\frac{1}{2} \mu_{3}-\mu_{4} \wp_{22}-4 \wp_{12}=0
$$

and expand the numerator in terms of $(\mathbf{u}-\tilde{\mathbf{u}})$, then we get a Taylor series in $\left(u_{1}-\tilde{u}_{1}\right)$ and $\left(u_{2}-\tilde{u}_{2}\right)$, equal to zero. Substituting in (A.1) and (A.1) gives an expansion in terms of the single parameter $\xi$. The relations between the $\sigma$ derivatives are then found by equating the coefficient of each power of $\xi$ with zero. Returning to equation (31), the coefficient of $\xi^{0}$ is

$$
8 \sigma_{2} \sigma_{222}-6{\sigma_{22}}^{2}-8 \sigma_{2} \sigma_{1}-2 \mu_{4}{\sigma_{2}}^{2}
$$

Setting this to zero we find

$$
\sigma_{222}=\frac{3}{4} \frac{\sigma_{22}^{2}}{\sigma_{2}}+\sigma_{1}+\frac{1}{4} \mu_{4} \sigma_{2}
$$

for any point on the divisor.

The following relations, calculated in the same way, are valid for $\mathbf{u} \in \Theta$ :

(from $(31))$;

$$
\begin{aligned}
\sigma_{111}= & -\frac{3}{4} \frac{{\sigma_{22}}^{2} \sigma_{1}^{3}}{\sigma_{2}{ }^{4}}-\frac{3}{2} \frac{\sigma_{22} \sigma_{1} \sigma_{11}}{\sigma_{2}^{2}}+\frac{3}{4} \mu_{4} \frac{\sigma_{1}{ }^{3}}{\sigma_{2}{ }^{2}}-3 \frac{\sigma_{1}{ }^{4}}{\sigma_{2}{ }^{3}}-\frac{3}{4} \mu_{3} \frac{\sigma_{1}^{2}}{\sigma_{2}}-3 \frac{\sigma_{1} \sigma_{12}^{2}}{\sigma_{2}^{2}} \\
& +3 \frac{\sigma_{12} \sigma_{22} \sigma_{1}^{2}}{\sigma_{2}{ }^{2}}+3 \frac{\sigma_{12} \sigma_{11}}{\sigma_{2}}+\mu_{2} \sigma_{1}-\frac{1}{2} \mu_{1} \sigma_{2}
\end{aligned}
$$

$($ from $(31))$;

$$
\begin{aligned}
\sigma_{112}= & \frac{1}{4} \frac{\sigma_{22}^{2} \sigma_{1}^{2}}{\sigma_{2}{ }^{3}}+\frac{1}{2} \frac{\sigma_{22} \sigma_{11}}{\sigma_{2}}-\frac{1}{4} \mu_{4} \frac{\sigma_{1}^{2}}{\sigma_{2}}+\frac{\sigma_{1}^{3}}{\sigma_{2}^{2}}+\frac{1}{4} \mu_{3} \sigma_{1} \\
& +\frac{\sigma_{12}^{2}}{\sigma_{2}}-\frac{\sigma_{22} \sigma_{1} \sigma_{12}}{\sigma_{2}^{2}}
\end{aligned}
$$

$$
\sigma_{122}=-\frac{1}{4} \frac{\sigma_{22}^{2} \sigma_{1}}{\sigma_{2}^{2}}-\frac{\sigma_{1}^{2}}{\sigma_{2}}+\frac{1}{4} \sigma_{1} \mu_{4}+\frac{\sigma_{12} \sigma_{22}}{\sigma_{2}}
$$

(also from (31)). An expression for the second order derivative $\sigma_{11}$ can also be obtained in this way. From the fifth PDE (31) we have

$$
\begin{aligned}
0= & \sigma_{11}^{2}+\left(2 \frac{\sigma_{22} \sigma_{1}^{2}}{\sigma_{2}^{2}}-4 \frac{\sigma_{1} \sigma_{12}}{\sigma_{2}}\right) \sigma_{11}+\left(\frac{\sigma_{1}^{4}{\sigma_{22}}^{2}}{\sigma_{2}{ }^{2}}+4 \frac{\sigma_{1}^{5}}{\sigma_{2}{ }^{3}}+\mu_{3} \frac{\sigma_{1}{ }^{3}}{\sigma_{2}}\right. \\
& \left.+4 \frac{\sigma_{1}^{2}{\sigma_{12}}^{2}}{\sigma_{2}{ }^{2}}-4 \frac{\sigma_{12} \sigma_{22} \sigma_{1}^{3}}{\sigma_{2}{ }^{3}}-\mu_{4} \frac{{\sigma_{1}}^{4}}{\sigma_{2}{ }^{2}}-\mu_{2}{\sigma_{1}}^{2}+\mu_{1} \sigma_{2} \sigma_{1}-\mu_{0} \sigma_{2}^{2}\right)
\end{aligned}
$$


and so

$$
\begin{aligned}
\sigma_{11}= & 2 \frac{\sigma_{1} \sigma_{12}}{\sigma_{2}}-\frac{\sigma_{22} \sigma_{1}{ }^{2}}{\sigma_{2}{ }^{2}} \\
& \pm \frac{1}{\sigma_{2}^{2}}\left(-4{\sigma_{1}}^{5} \sigma_{2}-\mu_{3} \sigma_{1}^{3} \sigma_{2}^{3}-\mu_{1} \sigma_{1} \sigma_{2}{ }^{5}\right. \\
& \left.+\mu_{4} \sigma_{1}{ }^{4}{\sigma_{2}}^{2}+\mu_{0}{\sigma_{2}}^{6}+\mu_{2}{\sigma_{1}}^{2} \sigma_{2}{ }^{4}\right)^{\frac{1}{2}}
\end{aligned}
$$

\section{Appendix B. Differential relations holding at $\mathbf{u}=\mathbf{u}_{0}$.}

At specific points $\mathbf{u}_{0}$, defined by $\sigma_{1}\left(\mathbf{u}_{0}\right)=0$, the first four identities in appendix Appendix A reduce to

$$
\begin{aligned}
\sigma_{111}\left(\mathbf{u}_{0}\right) & =3 \frac{\sigma_{12} \sigma_{11}}{\sigma_{2}}\left(\mathbf{u}_{0}\right)-\frac{1}{2} \mu_{1} \sigma_{2}\left(\mathbf{u}_{0}\right) \\
\sigma_{112}\left(\mathbf{u}_{0}\right) & =\frac{1}{2} \frac{\sigma_{22} \sigma_{11}}{\sigma_{2}}\left(\mathbf{u}_{0}\right)+\frac{\sigma_{12}^{2}}{\sigma_{2}}\left(\mathbf{u}_{0}\right) \\
\sigma_{122}\left(\mathbf{u}_{0}\right) & =\frac{\sigma_{12} \sigma_{22}}{\sigma_{2}}\left(\mathbf{u}_{0}\right)
\end{aligned}
$$

and

$$
\sigma_{222}\left(\mathbf{u}_{0}\right)=\frac{3}{4} \frac{\sigma_{22}^{2}}{\sigma_{2}}\left(\mathbf{u}_{0}\right)+\frac{1}{4} \mu_{4} \sigma_{2}\left(\mathbf{u}_{0}\right)
$$

Appendix C. Evaluation of $\zeta_{1}\left(2 \mathbf{u}_{0}\right)$.

The value of the constant $\zeta_{1}\left(2 \mathbf{u}_{\mathbf{0}}\right)$ may be calculated from the results of corollary 6.1 . From this we have

$$
2 \zeta_{1}\left(\mathbf{u}+\mathbf{u}_{\mathbf{0}}\right)=\frac{\sigma_{11}}{\sigma_{1}}(\mathbf{u})+\sqrt{\mu_{0}} \frac{\sigma_{2}}{\sigma_{1}}(\mathbf{u})+2 \frac{\sigma_{12}}{\sigma_{2}}\left(\mathbf{u}_{\mathbf{0}}\right) .
$$

Expanding the righthand side near $\mathbf{u}=\mathbf{u}_{0}$ we get that

$$
R H S=\left(\frac{1}{2} \frac{\mu_{1}}{\sqrt{\mu_{0}}}+4 \frac{\sigma_{12}}{\sigma_{2}}\left(\mathbf{u}_{0}\right)\right)+O\left(\left(u_{1}-u_{0,1}\right)\right)
$$

and so at the point $\mathbf{u}_{0}$

$$
\begin{aligned}
\left(\frac{1}{2} \frac{\mu_{1}}{\sqrt{\mu_{0}}}+4 \frac{\sigma_{12}}{\sigma_{2}}\left(\mathbf{u}_{0}\right)\right) & =2 \zeta_{1}\left(2 \mathbf{u}_{\mathbf{0}}\right) \\
& =\frac{\sigma_{11}}{\sigma_{1}}\left(\mathbf{u}_{0}\right)+\sqrt{\mu_{0}} \frac{\sigma_{2}}{\sigma_{1}}\left(\mathbf{u}_{0}\right)+2 \frac{\sigma_{12}}{\sigma_{2}}\left(\mathbf{u}_{\mathbf{0}}\right) \\
& =2 \frac{\sigma_{12}}{\sigma_{2}}\left(\mathbf{u}_{\mathbf{0}}\right)
\end{aligned}
$$

(since from $\left.(37) \sigma_{11}\left(\mathbf{u}_{0}\right)=-\sqrt{\mu_{0}} \sigma_{2}\left(\mathbf{u}_{0}\right)\right)$. Thus

$$
\zeta_{1}\left(2 \mathbf{u}_{\mathbf{0}}\right)=\frac{\sigma_{12}}{\sigma_{2}}\left(\mathbf{u}_{\mathbf{0}}\right)=-\frac{1}{4} \frac{\mu_{1}}{\sqrt{\mu_{0}}}
$$




\section{References}

[1] H F Baker 1907 Multiply Periodic Functions, (Cambridge: Cambridge Univ. Press)

[2] E D Belokolos, A I Bobenko, V Z Enolskii, A R Its and V B Matveev 1994 Algebro-Geometrical Approach to Nonlinear Integrable Equations (Berlin: Springer)

[3] D J Benney 1973 Some properties of long nonlinear waves Stud. Appl. Math $\mathbf{5 2} 45$

[4] V M Buchstaber, V Z Enolskii and D V Leykin Kleinian functions, hyperelliptic Jacobians and applications Reviews in Mathematics and Mathematical Physics 10:2 1

[5] B A Dubrovin and S P Novikov 1983 Hamiltonian formalism of one-dimensional systems of hydrodynamic type Soviet Math. Dokl. 27665

[6] V Z Enolskii, M Pronine and P H Richter 2003 Double pendulum and $\Theta$-divisor J. Nonlinear Science 13157

[7] E V Ferapontov and A P Fordy 1997 Separable Hamiltonians and integrable systems of hydrodynamic type J. of Geometry and Physics 21169

[8] J Gibbons 1981 Collisionless Boltzmann equations and integrable moment equations Physica D 3 503

[9] J Gibbons and S P Tsarev 1996 Reductions of the Benney equations Phys. Lett. A 21119

[10] J Gibbons and S P Tsarev 1999 Conformal maps and reductions of the Benney equations Phys. Lett. A 258263

[11] P Henrici 1974 Applied and computational Complex Analysis Volume 3 (New York: Wiley)

[12] B A Kupershmidt and Yu I Manin 1977 Long wave equation with a free surface I: Conservation laws and solutions Funct. Anal. Appl. 1131

[13] B A Kupershmidt and Yu I Manin 1978 Long wave equation with a free surface II: Hamiltonian structure and higher equations Funct. Anal. Appl. 1220

[14] S P Tsarev 1985 On Poisson Brackets and one-dimensional Hamiltonian systems of Hydrodynamic type Soviet Math. Dokl. 31488

[15] L Yu and J Gibbons 2000 The initial value problem for reductions of Lax equations Inverse Problems 16605

[16] V E Zakharov 1981 On the Benney equations Physica D 3 193;

V E Zakharov 1980 Funct. Anal. Appl. 1489 